\title{
Remoção de fármacos e desreguladores endócrinos em estações de tratamento de esgoto: revisão da literatura
}

\author{
Removal of pharmaceuticals and endocrine disrupters in \\ sewage treatment plants: literature review
}

\author{
Sérgio Francisco de Aquino \\ Professor adjunto Departamento de Química (DEQUI) da Universidade Federal de Ouro Preto (UFOP) - Ouro Preto (MG), Brasil. \\ Emanuel Manfred Freire Brandt \\ Doutorando do Programa de Pós-Graduação em Engenharia Sanitária e Ambiental pela Universidade \\ Federal de Minas Gerais (UFMG) - Belo Horizonte (MG), Brasil. \\ Carlos Augusto de Lemos Chernicharo \\ Professor associado Departamento de Engenharia Sanitária e Ambiental (DESA) da UFMG - Belo Horizonte(MG), Brasil.
}

\section{Resumo}

O monitoramento ambiental dos chamados microcontaminantes ou micropoluentes vem ganhando grande interesse da comunidade científica desde os anos 1970. Nesse grupo de contaminantes estão incluídos fármacos de diversas classes, produtos de limpeza e higiene pessoal, substâncias aplicadas na produção de plásticos e resinas, pesticidas, hormônios naturais e seus subprodutos, entre outros compostos. A principal via de contaminação do meio ambiente com fármacos e desreguladores endócrinos (DE) é o lançamento de esgotos in natura e tratado. Nesse contexto, este artigo compila dados de ocorrência de fármacos e DE no afluente e efluente de estações de tratamento de esgoto (ETE), discute os mecanismos envolvidos na sua remoção em ETE e faz uma análise comparativa da remoção de tais compostos em diferentes sistemas de tratamento.

Palavras-chave: microcontaminantes; esgoto; tratamento.

\begin{abstract}
The environmental monitoring of the so-called micropollutants has gained great interest since the 1970s. In this group of compounds are included several classes of pharmaceuticals, cleaning and personal care products, substances applied in plastics and resins, pesticides, natural hormones and their byproducts, among others. The main route of contamination of the environment with pharmaceuticals and endocrine disrupting compounds (EDC) is through the discharge of raw and treated sewage. Thus, this paper summarizes data on the occurrence of pharmaceuticals and EDC in raw and treated sewage, provides information regarding the mechanisms involved in their removal and compares their removal in different treatment processes.
\end{abstract}

Keywords: micropollutants; wastewater; treatment.

\section{Introdução}

O monitoramento no meio ambiente dos microcontaminantes vem recebendo grande interesse da comunidade científica desde o fim da década de 1970 (HIGNITE \& AZARNOFF, 1977; AHERNE; ENGLISH; MARKS,1985), especialmente devido ao reconhecimento dos seus efeitos, tais como: toxicidade aquática, genotoxicidade, perturbação endócrina em animais selvagens, seleção de bactérias patogênicas resistentes, entre outros (HALLING-SØRENSEN et al., 1998; KIM \& AGA, 2007; KÜMMERER, 2010). Nesse grupo de contaminantes estão incluídos os fármacos de diversas classes (ex: analgésicos; antibióticos; reguladores lipídicos; anti-inflamatórios; hormônios sintéticos), substâncias utilizadas em produtos de limpeza e higiene pessoal, compostos aplicados na produção de resinas e 
plásticos, além de hormônios naturais e outros. Os termos microcontaminantes ou micropoluentes também se aplicam a tais contaminantes emergentes e derivam da ocorrência de tais compostos no meio ambiente em concentrações da ordem de microgramas por litro $\left(\mu \mathrm{g} . \mathrm{L}^{-1}\right)$ ou inferiores.

Atenção especial vem sendo dada aos chamados desreguladores endócrinos (DE), devido às evidências de distúrbios causados por esses compostos no sistema reprodutivo de animais selvagens e até mesmo de seres humanos (CARLSEN et al., 1992; GUILLETTE et al., 1996; JOBLING et al., 1998; KASHIWADA et al., 2002; WHO, 2002; BILA \& DEZOTTI, 2007; VANDENBERG et al., 2007). Os DE podem ser classificados em (BILA \& DEZOTTI, 2007): (i) substâncias sintéticas denominadas xenoestrogênios, a exemplo dos alquilfenóis, pesticidas, ftalatos, bifenilas policloradas (PCB), bisfenol A e agentes farmacêuticos (ex.: 17 $\alpha$-etinilestradiol); (ii) substâncias naturais, a exemplo dos estrogênios (ex.: estrona e $17 \beta$-estradiol), androgênios (ex.: di-hidrotestosterona) e fitoestrogênios.

A tradução do termo endocrine disrupting chemicals tem gerado diversas outras denominações tais como (GHISELLI \& JARDIM, 2007): perturbadores endócrinos, disruptores endócrinos, agentes hormonalmente ativos e interferentes endócrinos. O termo "perturbador endócrino" tem origem na tradução exata da palavra "disrupt", que significa desfazer, perturbar ou interromper. O termo "disruptor endócrino" vem do aportuguesamento da palavra "disrupting" e não é definido pela língua portuguesa. O termo "agente hormonalmente ativo" é usado pelo fato de os compostos mimetizarem a ação de hormônios. Por sua vez, os termos "interferentes endócrinos" ou "desreguladores endócrinos" são usados para se referir a esses compostos, já que os mesmos interferem ou desregulam, de alguma forma, o funcionamento natural do sistema endócrino de espécies animais. Para efeito de consistência, neste artigo optou-se pelo uso do termo "desregulador endócrino", cujo significado foi julgado como mais adequado.

Os fármacos e DE podem atingir as redes de coleta de esgoto por meio do lançamento de águas cinzas (derivadas dos chuveiros, lavatórios e lavanderias), águas negras (excretas de indivíduos que podem conter medicamentos de uso oral e hormônios naturais) e descarte, nas instalações sanitárias, de medicamentos não usados ou com prazos de validade expirados. As estações de tratamento de esgoto (ETE) normalmente empregam processos biológicos como principal tecnologia e, em poucos casos, utilizam técnicas complementares de tratamento. Dessa forma, as unidades da ETE são projetadas para reduzir a carga de poluentes orgânicos e, eventualmente, nutrientes e microrganismos patogênicos, não objetivando especificamente a remoção de fármacos e DE presentes no esgoto sanitário. Qualquer remoção desses compostos que possa ocorrer é fortuita e inerente ao processo de tratamento (USEPA, 2009).

A revisão da literatura neste tópico mostra que diversos fármacos e DE permanecem praticamente incólumes ao tratamento de esgoto convencional, e várias pesquisas realizadas em diversos países mostram a ocorrência de fármacos em efluentes de ETE, corpos d'água, e, menos frequentemente, em água de consumo humano (CLARA et al. 2005b; KIM et al., 2007; MIÈGE et al., 2008; SODRÉ; LOCATELLI; JARDIM, 2010; TAMBOSI et al., 2010; JELIC et al., 2011). Ademais, no processo de tratamento de esgoto pode ocorrer o transporte de fármacos e DE da fase líquida para a sólida, constituindo em parte o resíduo sólido gerado nas ETE (lodo ou biomassa excedente) e potencializando os riscos relacionados à disposição final ou ao reúso do lodo na agricultura.

Segundo Suárez et al. (2008), o conhecimento das características dos microcontaminantes é muito importante para a elucidação dos mecanismos de degradação e transporte que ocorrem durante o tratamento de esgoto. Além disso, as condições operacionais e configurações das diversas unidades do tratamento podem influenciar nos mecanismos de sorção, fotodegradação, volatilização e transformações químicas e/ou biológicas dos compostos. Não obstante, são raras as pesquisas que relacionaram parâmetros operacionais e físico-químicos com a remoção dos fármacos e DE em ETE.

Considerando a importância do tema, este artigo tem como objetivo fornecer ao leitor informações a respeito dos mecanismos envolvidos na remoção de fármacos e DE em ETE. Complementarmente, este documento apresenta dados de ocorrência de alguns compostos relevantes (classificados como fármacos ou DE) em esgotos, efluentes de ETE e corpos d'água no Brasil e compara a remoção desses microcontaminantes em diferentes processos de tratamento de esgoto, com enfoque no efeito dos parâmetros operacionais sobre as eficiências de remoção.

\section{Ocorrência de fármacos e desreguladores endócrinos em amostras ambientais}

Os estudos acerca dos DE surgiram em consequência de eventos importantes que, a princípio, estiveram vinculados aos efeitos da exposição de seres humanos e animais a esses compostos. Importante acontecimento nesse aspecto foi a incidência de câncer no sistema reprodutivo de filhas de mulheres que usaram entre os anos de 1940 e 1970 o dietilestilbestrol, um agente terapêutico aplicado na gravidez para reduzir o risco de aborto e, atualmente, considerado um DE (BIRKETT \& LESTER, 2003 apud BILA \& DEZOTTI, 2007). Outro reconhecido estudo foi desenvolvido por Carlsen et al. (1992) na Dinamarca, em que se observou o declínio da qualidade do sêmen de homens entre os anos de 1938 e 1990, mas que, a despeito das supostas causas estarem relacionadas à exposição progressiva dos homens aos estrogênios ou compostos com atividade semelhante aos estrogênios, não foi objetivo do estudo confirmar essa hipótese.

Com relação à exposição de animais silvestres aos DE, um importante episódio foi observado no Lago Apopka na Flórida (EUA), em 1995, onde ocorreram anomalias no sistema reprodutivo de jacarés devido à contaminação com o pesticida DDT (2,2 bis-p-clorofenil-1,1,1-tricloroetano) e seu metabólito DDE (2,2 bis-p-clorofenil-1,1-dicloroetileno) (GUILLETTE et al., 1996). Ainda na década de 1990, no Reino Unido, 
foram observados diversos eventos de feminização de peixes devido à presença de compostos estrogênicos (hormônios naturais femininos ou substâncias que mimetizam estes), a exemplo do $17 \beta$-estradiol e dos alquilfenóis, presentes nos efluentes das ETE (PURDOM et al., 1994; JOBLING et al., 1998; SUMPTER, 1998).

Muitos outros problemas relacionados com os DE têm sido citados na literatura, entre os quais se destacam (BILA \& DEZOTTI, 2007): diminuição na eclosão de ovos de pássaros, peixes e tartarugas; problemas no sistema reprodutivo de répteis, pássaros e mamíferos; alterações no sistema imunológico de mamíferos marinhos. Em seres humanos, outros efeitos também são citados (BILA \& DEZOTTI, 2007), tais como: aumento da incidência de câncer de mama, de testículo e de próstata e a endometriose (doença caracterizada pela presença do tecido uterino fora do útero).

A partir de uma ampla revisão da literatura, Brandt (2012) concluiu que, a despeito das pesquisas realizadas até o momento indicarem alguns problemas relacionados à exposição de humanos e animais de laboratório a alguns $\mathrm{DE}$, não se pode afirmar que existem riscos à saúde pública. Isso se deve à escassez de evidências mais consistentes nos estudos toxicológicos existentes e à inexistência de estudos epidemiológicos que relacionem os níveis de exposição humana aos DE com os efeitos mencionados no parágrafo anterior. Por outro lado, as pesquisas sobre os efeitos causados pelos DE em ambientes aquáticos apontam resultados mais preocupantes. Em geral, essas pesquisas direcionam à seguinte conclusão (BRANDT, 2012): não existem evidências significativas de que as concentrações típicas dos DE existentes nos ambientes aquáticos possam causar efeitos agudos nos organismos aquáticos, mas existem estudos suficientes que apontam efeitos crônicos em organismos mais sensíveis submetidos a concentrações relevantes em termos de ocorrência dos compostos em ambientes aquáticos.

Além dos $\mathrm{DE}$, outros tipos de microcontaminantes (ex.: fármacos) têm sido tema de vários debates internacionais e pesquisas científicas. Como no caso dos DE, a divergência principal é se há evidências significativas de que tais compostos podem causar efeitos danosos em humanos e outros animais e se há níveis suficientes dessas substâncias no meio ambiente para gerar tais consequências. Outra questão em debate são os efeitos interativos causados pela mistura de diversos fármacos e DE no meio ambiente, implicando consequências inesperadas quando combinados, mesmo nas concentrações baixas encontradas no meio ambiente (POMATI et al., 2008; QUINN; GAGNÉ; BLAISE, 2009).

Atenção especial vem sendo dada aos antibióticos devido à possibilidade do desenvolvimento de bactérias patogênicas resistentes no meio ambiente. Ash e Iverson (2004) identificaram bactérias resistentes às sulfonamidas (um grupo de antibióticos) e à trimetoprima em rios dos EUA, fenômeno que parece ter ocorrido devido à presença desses compostos nos ambientes aquáticos, mesmo em baixas concentrações. Outro estudo realizado por Miranda e Castillo, (1998) apontou o desenvolvimento de resistência microbiana em uma espécie de
Aeromonas isolada de ambientes aquáticos após exposição a vários antibióticos (cloranfenicol, trimetoprima, sulfametoxazol, tetraciclina etc).

A literatura relata há várias décadas os efeitos causados pela exposição de diferentes organismos aos fármacos e DE. A partir do fim dos anos 1970, as pesquisas que avaliaram a ocorrência desses compostos no meio ambiente começaram a ser intensificadas, notadamente nos países europeus e nos EUA (HIGNITE \& AZARNOFF, 1977; WATTS et al., 1983 apud HALLING-SØRENSEN et al., 1998). Isso ocorreu devido ao desenvolvimento de novas técnicas analíticas (ex.: cromatografia de fase líquida acoplada à espectrometria de massas) que permitiram o monitoramento dessas substâncias nas baixas concentrações com que ocorrem no meio ambiente (IWA, 2010).

Na Figura 1 estão representadas as diferentes rotas de exposição humana aos fármacos e DE, destacando as principais matrizes ambientais em que esses compostos podem ser encontrados. No Brasil, as pesquisas que abordam a ocorrência de fármacos e DE nessas matrizes ambientais são esparsas. As Tabelas 1 e 2 apresentam os estudos acerca do tema no país até o momento, destacando, respectivamente, fármacos e DE presentes em esgoto sanitário, efluentes de ETE e águas superficiais. Para obter dados de estudos realizados em outros países sobre a ocorrência de fármacos e DE nessas matrizes ambientais, o leitor pode consultar Miège et al. (2008, 2009); Tambosi et al. (2010); IWA (2010); Brandt (2012), entre outros. Tanto os dados brasileiros quanto os estudos estrangeiros demonstram grande variabilidade na ocorrência dos fármacos e DE no esgoto sanitário bruto e tratado, o que possivelmente pode ser explicado pelo padrão de consumo diferenciado dessas substâncias. Diferentes países e regiões apresentam características bem distintas em termos de prevalência de doenças, de processos de tratamento de esgoto, de hábitos culturais ou até de restrições relacionadas ao mercado farmacêutico (ZUCCATO et al., 2006).

No caso dos fármacos, nas matrizes ambientais brasileiras (esgoto bruto, tratado e águas superficiais) observa-se ocorrência semelhante ou uma ordem de grandeza inferior às concentrações comumente relatadas na literatura estrangeira. Alguns anti-inflamatórios como o diclofenaco ou o ibuprofeno foram encontrados em concentrações maiores nos estudos de Ghiselli (2006) e Souza (2011), quando comparadas às concentrações comumente relatadas na literatura internacional. No caso dos hormônios naturais e sintéticos e dos xenoestrogênios, as concentrações encontradas nas matrizes ambientais brasileiras mostram-se semelhantes ou até três ordens de grandeza superiores às concentrações comumente relatadas na literatura internacional.

\section{Principais fármacos e desreguladores endócrinos abordados em estudos ambientais}

Os fármacos são compostos ativos complexos desenvolvidos e usados com o objetivo de promover efeitos biológicos específicos nos organismos. Após administradas, essas substâncias são absorvidas e 
distribuídas pelo corpo, parcialmente metabolizadas e finalmente excretadas do organismo (via fezes e urina) em suas formas originais, conjugadas ou como metabólitos (TAMBOSI, 2008). O metabolismo dos fármacos em um organismo inicia-se por diversas reações bioquímicas de hidroxilação, epoxidação, redução, hidrólise e adição de grupos funcionais. Em seguida, moléculas endógenas altamente polares ligam-se aos metabólitos formados nas reações anteriores ou aos próprios fármacos originais não-metabolizados (TAMBOSI et al., 2010), de forma a torná-los mais solúveis em água, facilitando assim a sua excreção. Entre as diversas moléculas endógenas responsáveis pelo processo de formação de conjugados, destacam-se os glicuronídeos, sulfatos e aminoácidos.

Ao contrário de outros microcontaminantes, tais como pesticidas e poluentes industriais, cujas concentrações no meio ambiente tenderão a diminuir nos próximos anos devido a restrições legais, é esperada uma ampliação no consumo dos fármacos em decorrência dos seus efeitos benéficos à saúde, podendo, consequentemente, resultar em aumento na sua ocorrência no meio ambiente (TAMBOSI et al., 2010).

De acordo com Santos et al. (2010), os fármacos mais frequentemente detectados em ambientes aquáticos são classificados como anti-inflamatórios não esteroides (16\%), antibióticos (15\%), reguladores lipídicos (12\%) e hormônios sintéticos (9\%), que somados perfazem 52\% dos 134 artigos publicados entre 1997 e 2009 sobre a ocorrência de fármacos em ambientes aquáticos. Por esse motivo, nesta revisão foram abordados apenas os principais fármacos representantes dessas classes.
O ibuprofeno (IBP) e o diclofenaco (DCF) são típicos representantes dos anti-inflamatórios mais citados na literatura, sendo abordado nesta revisão somente o DCF em função da sua elevada comercialização e prevalência ambiental. Já os antibióticos são caracterizados por uma grande variedade de classes, a exemplo das penicilinas, tetraciclinas, sulfonamidas e fluoroquinolonas. $\mathrm{Na}$ literatura existem dados para todas essas categorias, sendo mais frequentemente relatada a ocorrência de sulfametoxazol (SMX), trimetoprima (TRI) e tetraciclina (TET). Os dois primeiros antibióticos são normalmente estudados em conjunto por constituírem o princípio ativo do medicamento Bactrim ${ }^{\circledR}$, de grande utilização no nosso país, sendo por isso considerados nesta revisão. Entre os reguladores lipídicos (medicamentos utilizados para diminuir os níveis de colesterol e triglicérides no sangue), o bezafibrato (BZF) é o medicamento mais relatado na literatura. A principal referência a hormônios sintéticos recai sobre o $17 \alpha$-etinilestradiol ou etinilestradiol (EE2), fármaco amplamente utilizado em pílulas anticoncepcionais.

Com relação aos DE, destacam-se na literatura aqueles chamados de estrogênios, hormônios responsáveis pelo desenvolvimento das características femininas em um organismo. Entre suas principais funções destacam-se regulação do ciclo menstrual, estímulo ao desenvolvimento do endométrio (membrana mucosa que reveste a parede uterina) e dos seios e influência sobre o desenvolvimento e comportamento do organismo como um todo (NASSIF et al., 2005).

Estrogênios naturais, como estrona (E1), 17ß-estradiol ou estradiol (E2) e estriol (E3), são diariamente produzidos e excretados

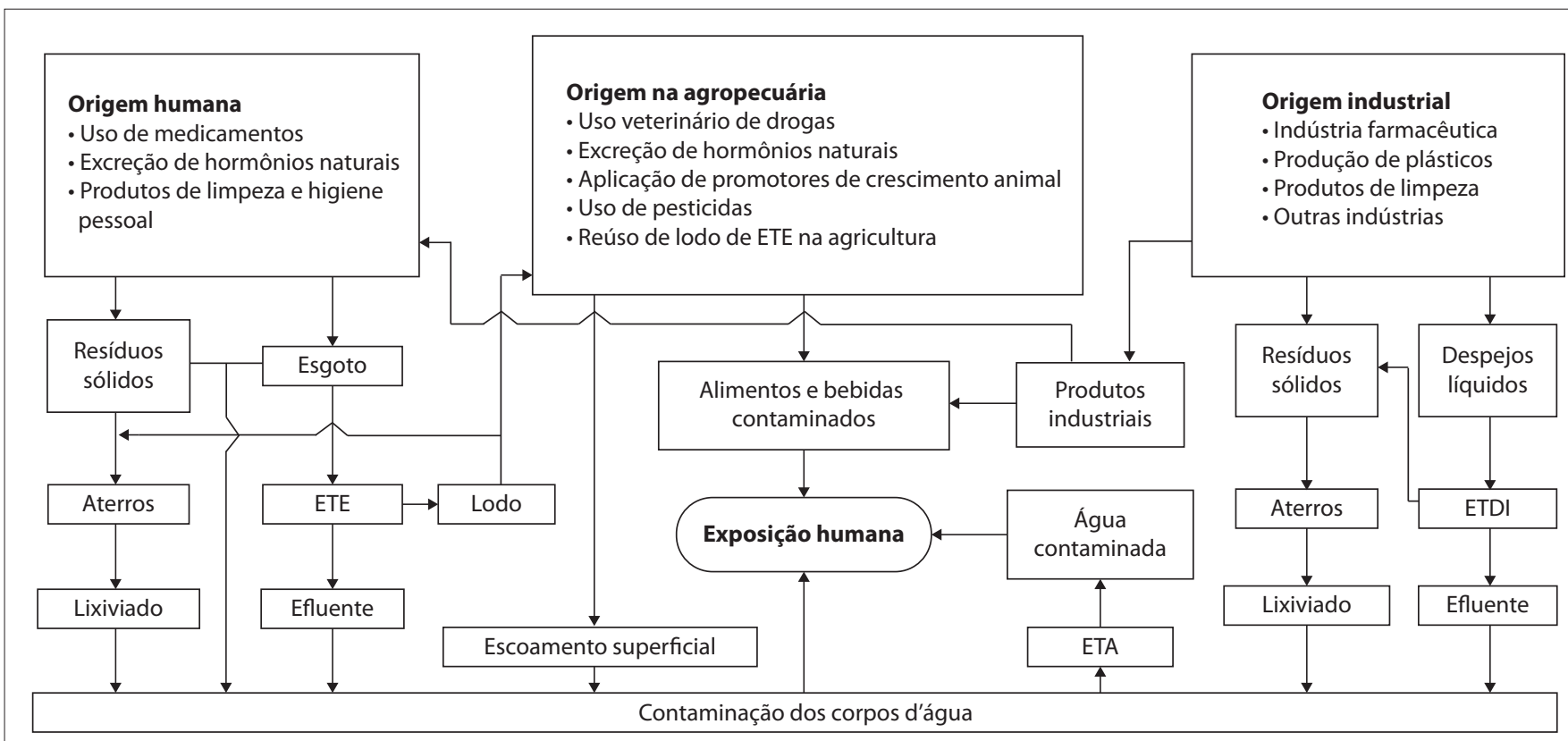

ETE e ETDI: Estações de Tratamento de Esgoto e de Despejos Industriais; ETA: Estação de Tratamento de Água

Figura 1 - Rotas de contaminação e exposição humana aos fármacos e desreguladores endócrinos. 
Tabela 1 - Estudos brasileiros acerca da ocorrência de fármacos em diferentes matrizes ambientais.

\begin{tabular}{|c|c|c|c|c|c|}
\hline \multirow{2}{*}{$\begin{array}{l}\mathbb{D} \\
\mathscr{W} \\
0 \\
\frac{\pi}{U}\end{array}$} & \multirow{2}{*}{$\begin{array}{l}\text { 옹 } \\
\text { 을 } \\
\text { ㅇํㅇ }\end{array}$} & \multicolumn{3}{|c|}{ 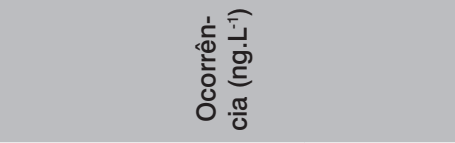 } & \multirow{2}{*}{ 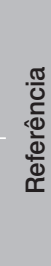 } \\
\hline & & 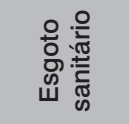 & 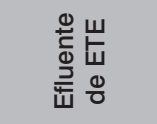 & 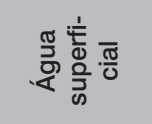 & \\
\hline \multirow{5}{*}{$\begin{array}{l}\text { Analgési- } \\
\text { cos }\end{array}$} & \multirow{2}{*}{$\begin{array}{l}\text { Ác. acetilsali- } \\
\text { cílico }\end{array}$} & - & $<50$ & - & $\begin{array}{c}\text { (1) } \\
a\end{array}$ \\
\hline & & - & - & $\begin{array}{c}<49- \\
20.960\end{array}$ & $\begin{array}{c}(2) \\
\mathrm{b}\end{array}$ \\
\hline & \multirow[b]{2}{*}{ Dipirona } & 36.400 & 23.700 & - & $\begin{array}{c}\text { (3) } \\
\mathrm{b}\end{array}$ \\
\hline & & $\begin{array}{c}<2.200- \\
8.970\end{array}$ & $\begin{array}{c}<2.200- \\
1.380\end{array}$ & - & $\begin{array}{c}(4) \\
c\end{array}$ \\
\hline & Paracetamol & 18.100 & 59.000 & $<8.300$ & $\begin{array}{c}\text { (3) } \\
\mathrm{b}\end{array}$ \\
\hline \multirow{8}{*}{$\begin{array}{l}\text { Antibióti- } \\
\text { cos }\end{array}$} & \multirow{3}{*}{$\begin{array}{l}\text { Sulfameto- } \\
\text { xazol }\end{array}$} & - & - & $<0,2-106$ & $\begin{array}{l}\text { (5) } \\
\mathrm{b}\end{array}$ \\
\hline & & - & $<1,9-124$ & - & (6) \\
\hline & & $<1,9-151$ & $<1,9-161$ & - & $\begin{array}{c}(7) \\
d\end{array}$ \\
\hline & \multirow{3}{*}{ Trimetoprima } & - & - & $<0,3-484$ & $\begin{array}{c}\text { (5) } \\
\mathrm{b}\end{array}$ \\
\hline & & - & $<1-75$ & - & $\begin{array}{l}\text { (6) } \\
d\end{array}$ \\
\hline & & 23-114 & $<1-88$ & - & $\begin{array}{c}(7) \\
d\end{array}$ \\
\hline & Amoxicilina & - & - & $<0,1-1.284$ & $\begin{array}{c}\text { (5) } \\
\mathrm{b}\end{array}$ \\
\hline & Tetraciclina & - & - & $<0,8-11$ & $\begin{array}{c}\text { (5) } \\
\mathrm{b}\end{array}$ \\
\hline \multirow{11}{*}{$\begin{array}{l}\text { Anti-infla- } \\
\text { matórios }\end{array}$} & Cetoprofeno & 570 & 150-290 & $<10$ & $\begin{array}{c}(1) \\
a\end{array}$ \\
\hline & \multirow{6}{*}{ Diclofenaco } & 790 & $200-700$ & $<10-60$ & $\begin{array}{c}(1) \\
a\end{array}$ \\
\hline & & - & - & $<14-115$ & $\begin{array}{c}\text { (2) } \\
\mathrm{b}\end{array}$ \\
\hline & & 2.870 & 1.780 & $\begin{array}{c}2.000- \\
6.000\end{array}$ & $\begin{array}{l}\text { (3) } \\
\mathrm{b}\end{array}$ \\
\hline & & $\begin{array}{l}<400- \\
5.600\end{array}$ & $<400-1.900$ & - & $\begin{array}{c}(4) \\
c\end{array}$ \\
\hline & & - & $<7-755$ & - & $\begin{array}{c}\text { (6) } \\
d\end{array}$ \\
\hline & & $<7-240$ & $<7-486$ & - & $\begin{array}{c}(7) \\
d\end{array}$ \\
\hline & \multirow{3}{*}{ Ibuprofeno } & 350 & $70-600$ & $<10$ & $\begin{array}{c}(1) \\
a\end{array}$ \\
\hline & & - & - & $<51$ & $\begin{array}{c}(2) \\
b\end{array}$ \\
\hline & & 54.200 & 48.400 & $<22.300$ & $\begin{array}{c}\text { (3) } \\
\mathrm{b}\end{array}$ \\
\hline & Naproxeno & 600 & $120-600$ & $<10-50$ & $\begin{array}{c}\text { (1) } \\
\mathrm{a}\end{array}$ \\
\hline \multirow{4}{*}{$\begin{array}{l}\text { Regu- } \\
\text { ladores } \\
\text { lipídicos }\end{array}$} & \multirow{3}{*}{ Bezafibrato } & 1.180 & $580-1.050$ & $<25$ & $\begin{array}{c}\text { (1) } \\
a\end{array}$ \\
\hline & & - & $<4,3-309$ & - & $\begin{array}{c}\text { (6) } \\
d\end{array}$ \\
\hline & & $<4,3-249$ & $<4,3-278$ & - & $\begin{array}{c}(7) \\
d\end{array}$ \\
\hline & Genfibrozil & 300 & $180-400$ & - & $\begin{array}{c}(1) \\
a\end{array}$ \\
\hline
\end{tabular}

(1) Stumpf et al., 1999; (2) Montagner e Jardim, 2011; (3) Ghiselli, 2006; (4) Souza, 2011; (5) Locatelli et al., 2011; (6) Brandt, 2012; (7) Queiroz et al., 2012.

aRio de Janeiro, Niterói, Resende, Três Rios e Campos (RJ); ' ${ }^{2} A t i b a i a$, Campinas, Paulínia e Americana (SP); ' Fortaleza (CE); 'Belo Horizonte (MG)
Tabela 2 - Estudos brasileiros acerca da ocorrência de desreguladores endócrinos em diferentes matrizes ambientais.

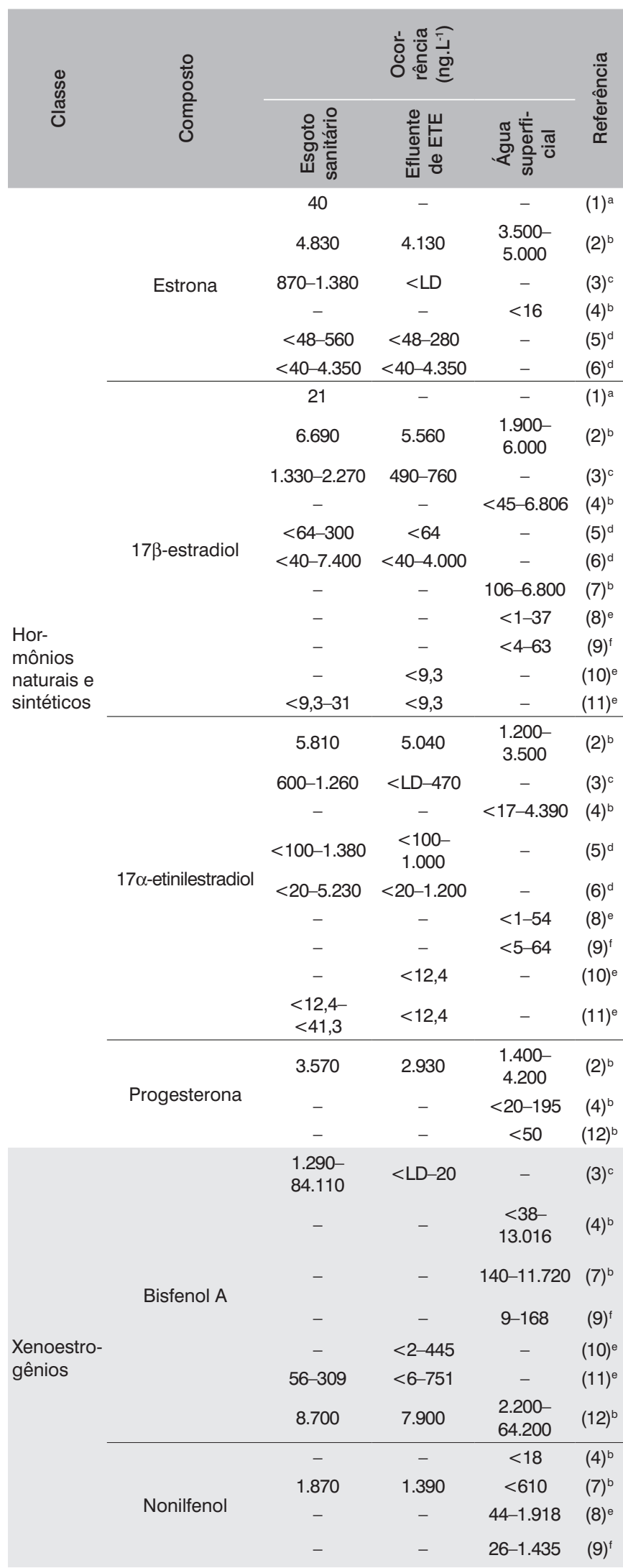

(1) Ternes et al., 1999; (2) Ghiselli, 2006; (3) Froehner, 2011; (4) Montagner e Jardim, 2011; (5) Pessoa et al., 2011; (6) Souza, 2011; (7) Montagner, 2007; (8) Moreira et al., 2009; (9) Moreira et al., 2011; (10) Brandt, 2012; (11) Queiroz et al., 2012; (12) Sodré, Locatelli e Jardim, 2010. aRio de Janeiro (RJ); ' ${ }^{2}$ Atibaia, Campinas, Paulínia e Americana (SP); ${ }^{\circ}$ Curitiba

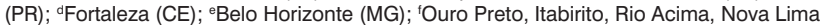
(MG). LD: limite de detecção 
pelas mulheres e animais vertebrados fêmeas (RIBEIRO et al., 2010). O EE2 também é um estrogênio, porém sintético, que apresenta maior atividade de desregulação endócrina do que os hormônios naturais (SVENSON et al., 2003).

Além dos estrogênios, outras substâncias usadas ou produzidas na indústria, tais como pesticidas, ftalatos, alquilfenóis, bifenilas policloradas e hidrocarbonetos policíclicos aromáticos, têm chamado a atenção da comunidade científica pela capacidade estrogênica aliada à constante presença em amostras de águas naturais e esgoto (bruto e tratado). Tais substâncias sintéticas competem com o E2 pelos receptores de estrogênio e podem exercer efeito sobre um organismo (GHISELLI \& JARDIM, 2007), o que as caracteriza como xenoestrogênios (o prefixo xeno- tem origem grega e significa estranho, estrangeiro). Dois xenoestrogênios amplamente encontrados em matrizes ambientais e de particular interesse nesta revisão são bisfenol A (BPA) e nonilfenol (NP).

O BPA é uma substância utilizada como monômero na produção de polímeros do tipo policarbonato, resinas epóxi e resinas de poliéster-estireno (STAPLES et al., 1998). É também usado como antioxidante em alguns plastificantes e como inibidor da polimerização no PVC. O BPA pode ser de 10.000 a 100.000 vezes menos potente do que o estradiol (VANDENBERG et al., 2007), sendo que a exposição humana a este composto ocorre principalmente pela ingestão direta de alimentos e bebidas contaminados. A dose estimada de exposição por ingestão direta está na faixa de $<1$ a $5 \mu \mathrm{g}$. $\mathrm{kg}^{-1}$. $\mathrm{dia}^{-1}$ (VANDENBERG et al., 2007).

Já o NP é um alquilfenol (AP) utilizado na produção dos nonilfenóis etoxilados (NPEO), surfactantes não iônicos amplamente utilizados como detergentes, emulsionantes, agentes umectantes e dispersantes (SOARES et al., 2008). Além de insumo, o NP é também subproduto da biodegradação dos NPEO, sendo essa a principal via de contaminação ambiental de tal microcontaminante (LANGFORD \& LESTER, 2002 apud SOARES et al., 2008). A biodegradação de alquilfenóis polietoxilados (APEO) pode ocorrer tanto em condições aeróbias, quanto em condições anaeróbias ou anóxicas, o que normalmente resulta na produção de mistura de metabólitos mais persistentes e estrogênicos, constituída de alquilfenóis mono a trietoxilados $\left(\mathrm{AP}_{1} \mathrm{EO}\right.$, $\mathrm{AP}_{2} \mathrm{EO}$ e $\mathrm{AP}_{3} \mathrm{EO}$ ), alquilfenóis mono a tricarboxilados ( $\mathrm{AP}_{1} \mathrm{EC}, \mathrm{AP}_{2} \mathrm{EC}$ e $\mathrm{AP}_{3} \mathrm{EC}$ ) e compostos fenólicos não etoxilados ( $\mathrm{AP}$ ), entre os quais se destacam os isômeros de NP (MAKI; FUJITA; FUJIWARA, 1996; EJLERTSSON et al., 1999; MANZANO et al., 1999).

\section{Fatores intervenientes na remoção de fármacos e desreguladores em estações de tratamento de esgoto}

Para se entender o destino dos fármacos e DE em uma ETE, deve-se avaliar os principais mecanismos de remoção atuantes, que por sua vez são definidos pelas propriedades físico-químicas dos microcontaminantes, pela configuração dos sistemas de tratamento, pelas condições ambientais e pelos parâmetros operacionais das unidades de tratamento (IWA, 2010). Os possíveis mecanismos de remoção de fármacos e desreguladores em ETE são detalhados a seguir.

\section{Sorção}

O termo sorção refere-se ao fenômeno de transferência de massa no qual moléculas passam de uma fase fluida (líquida ou gasosa) e tornam-se associados a uma fase sólida ou líquida. A transferência de massa na sorção de compostos presentes em meio aquoso pode ser estimada pelo coeficiente de distribuição ou partição sólido-líquido $\left(\mathrm{K}_{\mathrm{d}}\right)$, definido como a relação entre as concentrações de uma substância nas fases líquida e sólida em condições de equilíbrio (SUÁREZ et al., 2008). No caso dos mecanismos envolvidos na sorção de alguns fármacos e DE durante o tratamento de águas residuárias em uma ETE, pode-se dizer que, para um composto $i$ em condições de equilíbrio entre as fases líquida e sólida, a concentração na fase sólida $\left(C_{i, S}\right)$ ou a massa de $i$ por $\mathrm{kg}$ de sólidos suspensos $\left(\mathrm{C}_{\mathrm{i}, \mathrm{S}} \cdot \mathrm{SS}^{-1}\right)$ é proporcional à concentração do composto $i$ na fase líquida $\left(C_{i, I}\right)$, conforme mostra a Equação 1 (TERNES et al., 2004):

$C_{i, s}=K_{d} S S \cdot C_{i, L}$

na qual:

$\mathrm{C}_{\mathrm{i}, \mathrm{S}}$ é a concentração do composto i na fase sólida (ng. $\mathrm{L}^{-1}$ a $\left.\mu \mathrm{g} . \mathrm{L}^{-1}\right)$;

$\mathrm{K}_{\mathrm{d}}$ é o coeficiente de distribuição sólido-líquido $\left(\mathrm{L}_{\mathrm{kg}} \mathrm{kg}^{-1}\right)$;

SS é a concentração de sólidos suspensos no esgoto, podendo ser ainda a produção de lodo por litro de esgoto tratado $\left(\mathrm{kg} \cdot \mathrm{L}^{-1}\right)$;

$C_{i, L}$ é a concentração do composto $i$ dissolvido na fase líquida (ng. $\mathrm{L}^{-1}$ a $\left.\mu g . L^{-1}\right)$.

Nos estudos de balanço de massa de fármacos e DE em sistemas de tratamento de esgoto na modalidade lodos ativados, normalmente se desconsidera a liberação de sítios de adsorção nos sólidos pelo processo de biodegradação (sobre a fase sólida ou no meio líquido), assumindo-se que apenas os sólidos ou biomassa recém-gerada estão disponíveis para o processo de sorção dos compostos que adentram continuamente o reator biológico (TERNES et al., 2004). Contudo, tal premissa é uma simplificação do balanço de massa e pode ser aplicada a sistema de lodos ativados em função do tempo de retenção dos sólidos na unidade de tratamento ser muito superior ao tempo de detenção hidráulica do líquido (TDH) e da geração de sólidos ou biomassa no sistema ser elevada quando comparada à renovação dos sítios de sorção. Nesse caso, o termo SS não é a concentração de sólidos totais (ST) no esgoto ou no lodo, mas sim a quantidade de lodo ou biomassa gerada por unidade de esgoto tratado.

$\mathrm{O}$ valor de $\mathrm{K}_{\mathrm{d}}$ depende das características do poluente, assim como do tipo de lodo (primário, aeróbio, anaeróbio etc.) ou sólidos 
suspensos em questão (TERNES et al., 2004; SUÁREZ et al., 2008). O $\mathrm{K}_{\mathrm{d}}$ também está diretamente relacionando aos processos de adsorção e absorção. A adsorção refere-se ao fenômeno de transferência de massa na interface entre as fases, ao passo que a absorção ocorre quando moléculas penetram na fase sólida ou líquida além da interface.

No caso dos fármacos e DE em um sistema de tratamento biológico, a absorção refere-se às interações dos grupos alifáticos e aromáticos dos poluentes presentes na fase líquida com a membrana celular lipofílica dos microrganismos presentes na biomassa (lodo) ou com as frações lipídicas constituintes nos sólidos suspensos (SUÁREZ et al., 2008). Portanto, a lipofilicidade ou hidrofobicidade é a principal propriedade de um fármaco ou DE que determina a sua capacidade de absorção nas matrizes sólidas presentes em uma ETE, sendo estimada pelo coeficiente de partição octanol/água $\left(\mathrm{K}_{\mathrm{ow}}\right)$.

Fármacos e DE que apresentam log $\mathrm{K}_{\mathrm{ow}}<2,5$ são caracterizados por alta hidrofilicidade e baixa tendência de absorção na biomassa e nas frações lipídicas dos sólidos suspensos. Para aqueles que apresentam o $\log \mathrm{K}_{\mathrm{ow}}$ entre 2,5 e 4,0, espera-se uma tendência moderada de absorção nessas matrizes. Por sua vez, os fármacos e DE com $\log \mathrm{K}_{\mathrm{ow}}>4,0$ são altamente hidrofóbicos e têm um grande potencial de serem encontrados sorvidos nos sólidos presentes nos sistemas de tratamento de esgoto (ROGERS, 1996; TER LAAK et al., 2005).

A adsorção refere-se às interações eletrostáticas dos grupos positivamente carregados dos compostos com as superfícies carregadas negativamente da biomassa ou às fracas forças de interação do tipo Van der Waals entre grupos alifáticos dos fármacos e DE e as superfícies hidrofóbicas da biomassa e da matéria orgânica em suspensão. Para efeito prático, as forças de Van der Waals são normalmente desconsideradas por serem mais fracas do que as interações eletrostáticas. Por outro lado, tais forças são determinantes nos processos de absorção, conforme já mencionado.

Portanto, a adsorção está mais relacionada com a capacidade do fármaco ou do DE estar ionizado ou dissociado na fase aquosa, que por sua vez depende do pH do meio e, no caso de ácidos ou bases, da constante de acidez $\left(\mathrm{K}_{\mathrm{a}}\right)$ ou basicidade $\left(\mathrm{K}_{\mathrm{b}}\right)$ do composto. Moléculas poluentes básicas se ionizam no esgoto sanitário gerando cargas positivas e, portanto, tenderão a sofrer atração pela biomassa, que geralmente encontra-se carregada negativamente (SUÁREZ et al., 2008). Entretanto, há que se considerar que os sólidos suspensos também podem adquirir carga positiva ou neutra em função do $\mathrm{pH}$ do meio. $\mathrm{O}$ valor do ponto de carga zero ( $\mathrm{PCZ}$ - pH em que a soma de cargas é zero) ou do potencial zeta (potencial no plano hidrodinâmico de cisalhamento) indicam esse efeito.

Avanços recentes na metodologia analítica permitiram a determinação das concentrações de fármacos e DE sorvidos nos sólidos e alguns valores de $K_{d}$ já foram relatados na literatura (TERNES et al., 2004; CARBALLA et al., 2008; SUÁREZ et al., 2008). Contudo, o $\mathrm{K}_{\mathrm{d}}$ não depende somente das propriedades da molécula poluente, mas também das características do sólido em questão. Dessa forma, é necessário que se determine o $\mathrm{K}_{\mathrm{d}}$ para cada tipo de matriz encontrada nos diversos tipos de sistemas de tratamento de esgoto. Em geral, valores de $\log \mathrm{K}_{\mathrm{d}} \leq 2,0$ indicam tendência quase nula para a sorção do fármaco ou DE (TERNES et al., 2004). Já a faixa de $\log K_{d}$ compreendida entre 2,0 e 2,7 indica baixa tendência para a sorção do composto (TERNES et al., 2004), e valores superiores a 2,7 demonstram alta tendência do contaminante de sorção em uma matriz (TERNES et al., 2004).

A revisão da literatura mostra que, até o momento, valores do $\mathrm{K}_{\mathrm{d}}$ têm sido relatados apenas para as matrizes sólidas presentes em sistemas de lodos ativados, incluindo os lodos de decantadores primários, de tanques de aeração e de digestores anaeróbios. A Tabela 3 apresenta valores de $\mathrm{K}_{\mathrm{d}}$ para alguns dos compostos-alvo desta revisão. Ainda são necessários mais estudos para se determinar o $K_{d}$ em outras matrizes sólidas presentes nos diversos sistemas de tratamento de esgoto, tais como reatores anaeróbios (ex.: reatores UASB - Upflow Anaerobic Sludge Blanket), sistemas naturais de tratamento (ex.: lagoas de estabilização), reatores aeróbios com biofilmes (ex.: filtros percoladores), entre outros.

Além do $\mathrm{K}_{\mathrm{d}}$, existe outra variável indicadora do processo de sorção, chamada coeficiente de adsorção normalizado em função do conteúdo orgânico $\left(\mathrm{K}_{\mathrm{oc}}\right)$, que é mais conveniente para se estimar a porcentagem de uma substância adsorvida em uma matriz sólida no tratamento de esgoto. $\mathrm{O} \mathrm{K}_{\mathrm{oc}}$ pode ser calculado a partir do $\mathrm{K}_{\mathrm{d}} \mathrm{e}$ da fração orgânica ( $f$ oc nos sólidos, conforme mostra a Equação 2 (CARBALLA et al., 2008)

$K_{o c}=\frac{K_{d}}{f_{o c}}=\frac{K_{d}}{\% C O} \times 100$

na qual:

$\mathrm{K}_{\mathrm{oc}}$ é o coeficiente de adsorção normalizado em função do conteúdo

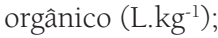

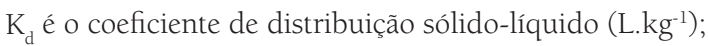

$\mathrm{f}_{\mathrm{oc}}$ é a fração orgânica da matriz sólida;

\%CO representa o percentual de carbono orgânico da matriz sólida.

Tabela 3 - Logaritmo do coeficiente de distribuição sólido-líquido ( $\left.\log \mathrm{K}_{\mathrm{d}}\right)$ de alguns fármacos e desreguladores endócrinos relevantes presentes em matrizes sólidas em sistema de lodos ativados.

\begin{tabular}{lccc} 
Composto & $\begin{array}{c}\text { Lodo primário } \\
\text { (decantador } \\
\text { primário) }\end{array}$ & $\begin{array}{c}\text { Lodo secun- } \\
\text { dário (lodos } \\
\text { ativados) }\end{array}$ & $\begin{array}{c}\text { Lodo digerido } \\
\text { (digestores } \\
\text { anaeróbios) }\end{array}$ \\
\hline Estrona & 2,8 & 2,9 & - \\
$17 \beta$-estradiol & - & $2,4-3,0$ & 2,7 \\
$17 \alpha$-etinilestradiol & 2,4 & $2,5-3,0$ & 2,6 \\
\hline Diclofenaco & 2,7 & 1,2 & 1,8 \\
\hline Sulfametoxazol & 2,6 & $2,3-2,6$ & 1,4 \\
\hline Trimetoprima & - & 2,3 & -
\end{tabular}

Fonte: Clara et al., (2004); Ternes et al., (2004); Andersen et al., (2005); Göbel et al., (2005); Carballa, Omil e Lema, (2007); Carballa et al., (2008); Suárez et al., (2008). 
Por ser dependente do $\mathrm{K}_{\mathrm{d}}$ e, por consequência, do $\mathrm{pK}_{\mathrm{a}}$ e do $\log$ $\mathrm{K}_{\mathrm{ow}}$ o $\mathrm{K}_{\mathrm{oc}}$ é um coeficiente que caracteriza a adsorção referente às interações eletrostáticas e não eletrostáticas (ex.: forças de Van der Waals e ligações de hidrogênio) com as superfícies da matéria orgânica. Carballa et al. (2008) usaram digestores de lodo em escala de bancada para determinar o $\mathrm{K}_{\mathrm{oc}}$ de diversos fármacos e $\mathrm{DE}$ em lodo aeróbio digerido anaerobiamente. Contudo, assim como no caso do $\mathrm{K}_{\mathrm{d}}$, ainda são necessários mais estudos de determinação do $\mathrm{K}_{\text {oc }}$ para diferentes tipos de lodo, de forma que essa variável possa ser usada em balanços de massa de fármacos e DE em ETE.

Devido à pequena quantidade de dados disponíveis na literatura sobre os referidos coeficientes de sorção, são mais comuns estudos de balanço de massa em que as concentrações dos fármacos e DE nas matrizes sólidas são determinadas experimentalmente, ou apenas qualitativamente estimadas por meio dos valores de $\log \mathrm{K}_{\text {ow }}$ e $\mathrm{pK}_{\mathrm{a}}$, mais acessíveis para a maioria dos compostos. A Tabela 4 apresenta os valores de $\log \mathrm{K}_{\mathrm{ow}}$ e $\mathrm{pK}_{\mathrm{a}}$ para alguns fármacos e DE de interesse desta revisão, bem como uma estimativa qualitativa da tendência de sorção. Pelo fato da adsorção por interações eletrostáticas ser dependente do pH do meio e do caráter ácido/básico da molécula, a estimativa foi feita nesse caso para diferentes valores de $\mathrm{pH}$ do meio.

Vale salientar que muitos fármacos e DE têm grupos funcionais polares (ex.: grupos carboxílicos, aldeídos e aminas), que podem interagir com grupos específicos da matéria orgânica presentes nos sólidos. Portanto, em alguns casos os valores $\log \mathrm{K}_{\mathrm{ow}}$ e $\mathrm{pK}_{\mathrm{a}}$ são inadequados para se estimar os mecanismos de sorção nas matrizes sólidas presentes em ETE (TERNES et al., 2004). Nesses casos, os coeficientes $\mathrm{K}_{\mathrm{d}}$ e $\mathrm{K}_{\mathrm{oc}}$ são mais adequados por serem valores baseados em estudos experimentais.

No caso específico do SMX, pelo fato de a molécula desse antibiótico ter duas aminas ionizáveis, são verificados dois valores de $\mathrm{pK}_{\mathrm{a}}$. Como resultado, se o $\mathrm{pH}$ do meio estiver entre os valores do $\mathrm{pK}_{\mathrm{a}}$, o SMX estará presente como uma espécie predominantemente neutra. Se o pH do ambiente estiver abaixo ou acima do primeiro e do segundo $\mathrm{pK}_{\mathrm{a}}$, o SMX estará presente como uma espécie carregada positivamente ou negativamente, respectivamente.

\section{Transformações biológicas}

Embora a biodegradação seja uma das transformações mais importantes no tratamento de esgoto, no caso dos fármacos e dos $\mathrm{DE}$, o processo é limitado do ponto de vista cinético devido às baixas concentrações dos compostos verificadas no esgoto bruto. Segundo Joss et al. (2006), a maior parte desses microcontaminantes apresentam cinética de degradação de pseudo-primeira ordem, o que significa que a taxa de transformação biológica é diretamente proporcional à concentração dos compostos presentes na fase líquida, além da concentração da biomassa (expressa como sólidos suspensos - SS) no sistema, que é considerada em excesso e constante para fins de integração da Equação diferencial 3:

$\frac{d C_{i}}{d t}=K_{b i o} \cdot S S \cdot C_{i, L}$

na qual:

$\mathrm{C}_{\mathrm{i}}$ é a concentração total do composto $i$ (ng. $\mathrm{L}^{-1}$ a $\left.\mu g . \mathrm{L}^{-1}\right)$;

té o tempo (d);

$\mathrm{K}_{\mathrm{bio}}$ refere-se à constante de biodegradação do composto $i\left(\mathrm{~L}_{\mathrm{ss}}{ }^{-1} \cdot \mathrm{d}^{-1}\right)$; SS é a concentração de sólidos suspensos no lodo (g..-1 $)$;

$\mathrm{C}_{\mathrm{i}, \mathrm{L}}$ é a concentração do composto $i$ solúvel na fase líquida (ng. $\mathrm{L}^{-1}$ a $\left.\mu \mathrm{g} \cdot \mathrm{L}^{-1}\right)$.

Joss et al. (2006) realizaram experimentos para a determinação do $\mathrm{K}_{\mathrm{bio}}$ de vários microcontaminantes. A pesquisa foi realizada

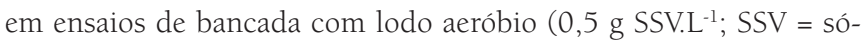
lidos suspensos voláteis) proveniente de um sistema de lodos ativados cujo tempo de retenção dos sólidos ou idade do lodo variava de 10 a 15 dias. Foram adicionados vários microcontaminantes em

Tabela 4 - Propriedades físico-químicas de alguns fármacos e desreguladores endócrinos relevantes e potencial para sorção em matrizes sólidas carregadas negativamente presentes em estações de tratamento de esgoto.

\begin{tabular}{|c|c|c|c|c|c|c|c|c|c|c|c|c|c|}
\hline \multirow{3}{*}{ Composto } & \multirow{3}{*}{ Abreviação } & \multirow{3}{*}{$\begin{array}{c}\text { Identificador } \\
\text { CAS }^{\mathrm{a}}\end{array}$} & \multirow{3}{*}{$\log \mathrm{K}_{\mathrm{ow}}$} & \multirow{3}{*}{$\mathrm{pK}_{\mathrm{a}}$} & \multicolumn{9}{|c|}{ Tendência para a sorção } \\
\hline & & & & & \multirow[t]{2}{*}{$\begin{array}{l}\text { Sorção } \\
\text { lipofílica }\end{array}$} & \multicolumn{8}{|c|}{ Adsorção por interações eletrostáticas } \\
\hline & & & & & & $\mathrm{pH}$ & 3 & 5 & 6 & 7 & 8 & 9 & 11 \\
\hline $17 \beta$-estradiol & E2 & $50-28-2$ & 4,01 & 10,4 & ++ & & l & 1 & 1 & l & l & l & -- \\
\hline $17 \alpha$-etinilestradiol & EE2 & $57-63-6$ & 3,67 & 10,4 & + & & l & l & I & l & l & l & -- \\
\hline Bisfenol A & BPA & $80-05-7$ & 3,32 & 10,2 & + & & I & I & I & l & l & l & -- \\
\hline Nonilfenol & NP & $25154-52-3$ & 5,71 & 10,3 & ++ & & I & 1 & 1 & 1 & 1 & 1 & - - \\
\hline Diclofenaco & DCF & $15307-86-5$ & 4,51 & 4,2 & ++ & & I & -- & -- & -- & -- & -- & -- \\
\hline Sulfametoxazol & SMX & $723-46-6$ & 0,89 & $1,6 / 5,7^{b}$ & -- & & I & - & -- & -- & -- & -- & - - \\
\hline Trimetoprima & TRI & $738-70-5$ & 0,91 & 7,12 & -- & & ++ & ++ & ++ & + & 1 & 1 & I \\
\hline Bezafibrato & BZF & 41859-67-0 & 4,32 & 3,3 & ++ & & - & -- & -- & - & - & - & - - \\
\hline
\end{tabular}

Fonte: TOXNET, acessado em setembro de 2011.

${ }^{a}$ CAS: Chemical Abstract Service. Número que corresponde à identificação química da substância; ${ }^{b}$ Primeiro/segundo pK ++ : grande tendência; + : tendência moderada; - : hidrofilicidade ou repulsão moderadas; - - : hidrofilicidade ou repulsão intensas; / : sem efeito 
concentrações similares àquelas usualmente observadas no esgoto sanitário $\left(3 \mu \mathrm{g} . \mathrm{L}^{-1}\right)$, sendo os valores de $\mathrm{K}_{\text {bio }}$ obtidos a partir das medidas de decaimento das concentrações dos microcontaminantes considerando-se modelo cinético de pseudo-primeira ordem. Alguns valores de $\mathrm{K}_{\text {bio }}$ de interesse particular para esta revisão são (JOSS et al., 2006): 550 a $950 \mathrm{~L} \cdot g S^{-1} \cdot \mathrm{d}^{-1}$ para o E2; 7 a $9 \mathrm{~L} \cdot g S^{-1} \cdot \mathrm{d}^{-1}$ para o EE2; $<0,1 \mathrm{~L} \cdot \mathrm{gSS}^{-1} \cdot \mathrm{d}^{-1}$ para o DCF; $<0,1$ a $0,3 \mathrm{~L} \cdot \mathrm{gSS}^{-1} \cdot \mathrm{d}^{-1}$ para o SMX; 4 a 7 L.gSS ${ }^{-1} \cdot d^{-1}$ no caso do BZF.

Joss et al.(2006) também concluíram que, no caso dos compostos com pouca tendência para a sorção na biomassa aeróbia $\left(\log K_{d} \leq 2,0\right)$, a divisão do volume reacional do reator em vários compartimentos (reator em regime hidráulico de fluxo em pistão) melhorou significativamente a remoção dos compostos biodegradáveis, quando comparado a um reator em regime hidráulico de mistura completa. O impacto positivo da aproximação do reator ao regime hidráulico de fluxo em pistão foi menos significativo para compostos com grande tendência para a sorção. Com base nesses resultados, Joss et al. (2006) propuseram a seguinte classificação dos fármacos e DE quanto ao grau de biodegradabilidade:

- Substâncias com $\mathrm{K}_{\text {bio }}<0,1 \mathrm{~L} \cdot g S^{-1} \cdot \mathrm{d}^{-1}$ não apresentam remoção satisfatória pelo mecanismo de biodegradação, sendo a eficiência máxima de remoção do composto na ETE inferior a 20\% para compostos com grande tendência para a sorção $\left(\log \mathrm{K}_{\mathrm{d}}>3\right)$;

- Substâncias com $0,1 \leq \mathrm{K}_{\mathrm{bio}} \leq 10 \mathrm{~L} \cdot \mathrm{gSS}^{-1} \cdot \mathrm{d}^{-1}$ devem ser parcialmente biodegradados com eficiência de remoção de 20 a 90\%;

- Para compostos com $\mathrm{K}_{\mathrm{bio}}>10$, espera-se uma boa remoção biológica (acima de 90\%), sendo a eficiência dependente do regime hidráulico do reator; são observadas maiores eficiências de remoções em reatores com fluxo hidráulico do tipo pistão.

Outro estudo realizado por Joss et al. (2004) demonstrou que a remoção biológica de alguns estrogênios foi claramente dependente da atividade da biomassa e do potencial redox nos sistemas de tratamento. Dessa forma, a concentração de oxigênio dissolvido no ambiente é um importante fator na remoção biológica dos fármacos e DE, notadamente para o caso dos DE, que são mais facilmente degradados em condições aeróbias quando comparadas às condições anaeróbias (IWA, 2010). A baixa biodegradabilidade anaeróbia dos DE provavelmente decorre da presença dos anéis aromáticos fenólicos em suas estruturas, que são mais dificilmente degradados na ausência de oxigênio dissolvido.

A despeito da importância do estabelecimento do $\mathrm{K}_{\text {bio }}$ para alguns fármacos e $\mathrm{DE}$ em diversos sistemas de tratamento sobre condições ambientais e operacionais variadas, nenhum estudo adicional foi encontrado na literatura, o que indica a necessidade do aprofundamento científico no tema. Como exemplo, não foram encontrados na literatura artigos sobre a remoção de fármacos e DE em sistemas anaeróbios ou anóxicos empregados na remoção biológica de nutrientes, como nitrogênio e fósforo.
Por outro lado, fatores que afetam a remoção biológica dos fármacos e DE em ETE são frequentemente relatados na literatura. A disponibilidade dos fármacos e DE para a assimilação pelos microrganismos é um dos fatores mais importantes na determinação da taxa de biodegradação em uma ETE (BURGESS et al., 2005). Em geral, a biodisponibilidade é definida pela combinação dos aspectos físico-químicos relacionados à distribuição e à transferência de massa dos compostos entre as fases líquida e sólida, e dos aspectos fisiológicos relacionados aos microrganismos. Uma alta biodisponibilidade depende principalmente da solubilidade do fármaco ou do DE em meio aquoso (CIRJA et al., 2008), sendo que compostos com $\log \mathrm{K}_{\mathrm{ow}}<2,5$ têm elevada hidrofilicidade e, portanto, elevada biodisponibilidade (IWA, 2010).

A biodisponibilidade depende também da atividade dos microrganismos e, dessa forma, a temperatura também é um fator importante, uma vez que o crescimento microbiano é determinado por faixas de temperaturas ótimas. Ademais, em temperaturas mais elevadas o processo de sorção, que é exotérmico, é desfavorecido e o composto apresentará uma maior solubilidade no meio aquoso, favorecendo ainda mais sua biodisponibilidade (IWA, 2010).

O TDH e o tempo de retenção dos sólidos no sistema (idade do lodo) são outros importantes fatores que influenciam na remoção biológica dos fármacos e DE em ETE. Na realidade, esses fatores também influenciam os mecanismos de sorção. Elevado TDH, assim como elevada idade do lodo permitem maior tempo para a biodegradação e para a sorção dos compostos dissolvidos.

Suárez et al. (2008) relacionaram as propriedades físico-químicas das moléculas, o TDH e a idade do lodo das unidades do sistema de tratamento biológico com a eficiência de remoção de microcontaminantes e generalizaram as seguintes conclusões:

- Compostos com elevados valores de $\mathrm{K}_{\text {bio }}$ e baixos valores de $\mathrm{K}_{\mathrm{d}}$ são muito bem biodegradados independente do TDH e da idade do lodo do sistema de tratamento;

- Compostos com baixos valores de $\mathrm{K}_{\text {bio }}$ e elevados valores de $\mathrm{K}_{\mathrm{d}}$ são retidos no sistema de tratamento por meio da sorção e biodegradados quando a idade do lodo é suficiente para permitir desorção e subsequente biodegradação;

- Compostos com elevados valores de $\mathrm{K}_{\mathrm{bio}}$ e valores de $\mathrm{K}_{\mathrm{d}}$ intermediários são moderadamente biodegradados independentemente do TDH. A remoção dessas substâncias é ligeiramente dependente da idade do lodo;

- Compostos com baixos valores de $\mathrm{K}_{\text {bio }}$ e $\mathrm{K}_{\mathrm{d}}$ não são removidos por sorção, nem biodegradados, independente do TDH e da idade do lodo.

Outros mecanismos de degradação, que não envolvem microrganismos, também podem ocorrer nas unidades de uma ETE. A degradação química pelo processo de hidrólise de substâncias conjugadas dos fármacos e DE é um exemplo de degradação abiótica. Contudo, a hidrólise química é desprezível na maior parte dos casos, já que os 
processos biológicos são muito mais importantes no fenômeno da desconjugação de fármacos e DE (CIRJA et al., 2008). Muitas vezes, a ação dos microrganismos na hidrólise de conjugados se inicia antes mesmo da entrada do esgoto na ETE (GOMES et al., 2009), nas redes de coleta de esgoto. Além disso, existe a possibilidade de remoção dos fármacos e dos DE por fotodegradação ou por volatilização, mecanismos discutidos a seguir.

\section{Fotodegradação}

A fotodegradação ou fotólise é um processo de degradação dos fármacos e DE que pode ocorrer de forma direta ou indireta. A fotodegradação direta é observada quando a molécula do microcontaminante tem uma ligação química que é clivada quando exposta e excitada pela radiação UV (GURR \& REINHARD, 2006). Na fotodegradação indireta, algumas moléculas presentes no meio são excitadas pela radiação UV da mesma forma como no mecanismo anterior e, após a clivagem dessas moléculas, são formados radicais livres que podem reagir com os microcontaminantes degradando-os (GURR \& REINHARD, 2006). Artigos recentes mostram que compostos dissolvidos, tais como a matéria orgânica natural (MON), podem atuar tanto como fotossensibilizadores ou como inibidores da oxidação de contaminantes aquáticos (WENK; Von GUNTEN; CANONICA, 2011).

$\mathrm{Na}$ maioria das vezes a fotodegradação natural (realizada pela radiação solar) é um mecanismo desprezível para a remoção de fármacos e DE em ETE convencionais, que empregam somente processos biológicos. Isso se deve principalmente às configurações das unidades de tratamento (IWA, 2010), à elevada presença de sólidos suspensos no esgoto (bruto e também tratado) e à pequena fração de radiação UV do espectro solar. Nos sistemas compactos, a área superficial disponível para a incidência solar é pequena, sendo restrita apenas às primeiras camadas da coluna de água em unidades abertas, a exemplo de decantadores e tanques de aeração (ZHANG; GEIBEN; GAL, 2008). Contudo, a fotodegradação pode ocorrer em maior monta em sistemas naturais (ex.: lagoas de estabilização), nos quais são empregadas grandes áreas superficiais para a incidência solar. O processo nesse caso pode ser limitado pelos períodos noturnos e pelos dias nublados, bem como pela presença de sólidos (ex.: algas) na fase líquida.

Não obstante, até o momento não foram encontrados na literatura estudos que objetivaram avaliar a contribuição da fotodegradação na remoção de fármacos e DE em ETE. As pesquisas no tema são restritas ao processo de fotodegradação que ocorre em águas superficiais ou em escala laboratorial com o uso de lâmpadas para simular a luz solar natural (BUSER; POIGER; MÜLLER, 1998; JÜRGENS et al., 2002; ANDREOZZI; RAFFAELE; NICKLAS, 2003; LEECH; SNYDER; WETZEL, 2009; CHOWDHURY; CHARPENTIER; RAY, 2010, 2011). Com base em tais estudos e considerando os compostos-alvo desta revisão, os principais fármacos e DE tidos como fotolábeis, ou seja, susceptíveis à fotodegradação natural, são: E2, EE2, DCF e SMX (BUSER; POIGER; MÜLLER, 1998; JÜRGENS et al., 2002; ANDREOZZI; RAFFAELE; NICKLAS, 2003).
Volatilização

A volatilização dos fármacos e dos DE em ETE pode ser estimada pela constante da Lei de Henry adimensional $\left(K_{H}^{\prime}\right)$, que determina o equilíbrio entre moléculas dissolvidas na fase líquida com aquelas presentes na fase gasosa em um sistema fechado. Tal processo é expressivo somente para compostos que apresentam $\mathrm{K}_{\mathrm{H}}^{\prime}>10^{-4}$ (STENSTROM et al., 1989). Além disso, o mecanismo de volatilização pode ser influenciado pelo $\mathrm{K}_{\mathrm{ow}}$, pois elevados valores desse coeficiente favorecem a retenção das substâncias nos sólidos em detrimento do processo de volatilização (ROGERS, 1996; GALASSI et al., 1997). Dessa forma, compostos que apresentam relação $\mathrm{K}_{\mathrm{H}} / \mathrm{K}_{\mathrm{ow}}<10^{-9}$ têm baixo potencial para a volatilização, mesmo quando o valor de $\mathrm{K}_{\mathrm{H}}^{\prime}$ é maior do que $10^{-4}$ (ROGERS, 1996). A Tabela 5 apresenta valores das constantes da Lei de Henry adimensionais e das relações $\mathrm{K}_{\mathrm{H}}^{\prime} / \mathrm{K}_{\mathrm{ow}}$ dos fármacos e dos $\mathrm{DE}$ de interesse particular desta revisão. Conforme depreende-se da Tabela 5, para nenhum dos compostos o mecanismo de volatilização deve ser expressivo.

A maioria dos fármacos e DE têm estruturas de elevada massa molar e, por consequência, são pouco voláteis. Por esse motivo, o mecanismo de remoção desses compostos em ETE via volatilização é desprezado em praticamente todas as pesquisas no tema. Mesmo em sistemas de tratamento aerados mecanicamente, nos quais pode ocorrer o desprendimento de substâncias para a fase gasosa, a remoção de fármacos e DE por volatilização é desprezível. Segundo Suárez et al. (2008), a possibilidade de desprendimento dos fármacos e dos DE em sistema de lodos ativados é praticamente nula, quando se leva em conta as taxas de aeração comumente aplicadas.

\section{Remoção de fármacos e desreguladores endócrinos em diferentes sistemas de tratamento}

A remoção dos fármacos e DE presentes no esgoto sanitário representa uma importante barreira no controle do aporte de microcontaminantes aos ambientes aquáticos. Algumas tecnologias disponíveis para a efetiva remoção desses compostos em ETE incluem (IWA, 2010): processos oxidativos avançados, ozonização, reatores

Tabela 5 - Constantes da Lei de Henry, coeficiente de partição octanol/água e relação $\mathrm{K}_{\mathrm{H}}^{\prime} / \mathrm{K}_{\mathrm{ow}}$ para alguns fármacos e desreguladores endócrinos relevantes.

\begin{tabular}{lccc}
$\begin{array}{l}\text { Compos- } \\
\text { to }\end{array}$ & $\begin{array}{c}\text { Constante da Lei de Henry } \\
\mathrm{K}_{\mathrm{H}}^{\prime}\end{array}$ & $\mathrm{K}_{\text {ow }}$ & $\mathrm{K}_{\mathrm{H}^{\prime}} / \mathrm{K}_{\text {ow }}$ \\
\hline E2 & $2,5 \times 10^{-10}$ & $1,0 \times 10^{4}$ & $2,5 \times 10^{-14}$ \\
\hline EE2 & $1,5 \times 10^{-10}$ & $4,7 \times 10^{3}$ & $3,1 \times 10^{-14}$ \\
\hline BPA & $4,1 \times 10^{-10}$ & $2,1 \times 10^{3}$ & $1,9 \times 10^{-13}$ \\
\hline NP & $4,5 \times 10^{-5}$ & $5,1 \times 10^{5}$ & $8,8 \times 10^{-11}$ \\
\hline DCF & $1,9 \times 10^{-10}$ & $3,2 \times 10^{4}$ & $5,9 \times 10^{-15}$ \\
SMX & $2,6 \times 10^{-11}$ & $0,8 \times 10^{1}$ & $3,3 \times 10^{-12}$ \\
\hline TRI & $9,8 \times 10^{-13}$ & $0,8 \times 10^{1}$ & $1,2 \times 10^{-13}$ \\
BZF & $8,7 \times 10^{-14}$ & $2,1 \times 10^{4}$ & $4,1 \times 10^{-18}$
\end{tabular}

Fonte: TOXNET, acessado em 2011; Mes et al. (2005); Suárez et al. (2008). 
com lâmpadas ultravioleta, processos de adsorção em carvão ativado. Contudo, esses processos demandam altos custos de investimento e apresentam operação mais onerosa e/ou sofisticada em relação aos sistemas biológicos de tratamento.

Considerando que a maior parte das ETE não emprega tais tecnologias, torna-se importante avaliar a remoção de fármacos e $\mathrm{DE}$ em sistemas de tratamento de esgoto convencionais, que utilizam reatores biológicos. Nesse aspecto, existem diversos estudos que avaliaram a remoção de fármacos e DE em sistemas de lodos ativados (CLARA et al., 2005a; MIĖGE et al., 2008; SIPMA et al., 2009).

\section{Lodos ativados}

A Figura 2 ilustra informações de eficiências de remoção de alguns fármacos e DE em sistemas de tratamento de esgoto na modalidade lodos ativados, compiladas de vários estudos: Carballa et al. (2004), Clara et al. (2005a, 2005b), Joss et al. (2005), Vieno, Tuhkanen, e Kronberg (2005), Batt, Kim, e Aga (2006), Bernhard, Müller e Thomas (2006), Lishman et al. (2006), Nakada et al. (2006), Göbel et al. (2007), Radjenovic, Petrovic e Barceló (2007; 2009), Tan et al. (2007), Gulkowska et al. (2008), Pothitou e Voutsa (2008), Ying, Kookana e Kumar (2008), Janex-Habib et al. (2009), Kasprzyk-Hordern, Dinsdale e Guwy, (2009), Choubert et al. (2011), Jelic et al. (2011), McAdam et al. (2011), Pedrouzo et al. (2011), Zhang et al. (2011).

Para se eliminar o efeito das condições operacionais diferenciadas verificadas em cada estudo, os dados encontrados na literatura foram segregados de acordo com o tempo de retenção dos sólidos nos sistemas de tratamento (idade do lodo). Optou-se pela comparação da idade do lodo de 10 dias pelo fato de ser esse um valor crítico para a remoção dos fármacos e DE (SAIANO et al., 2004;

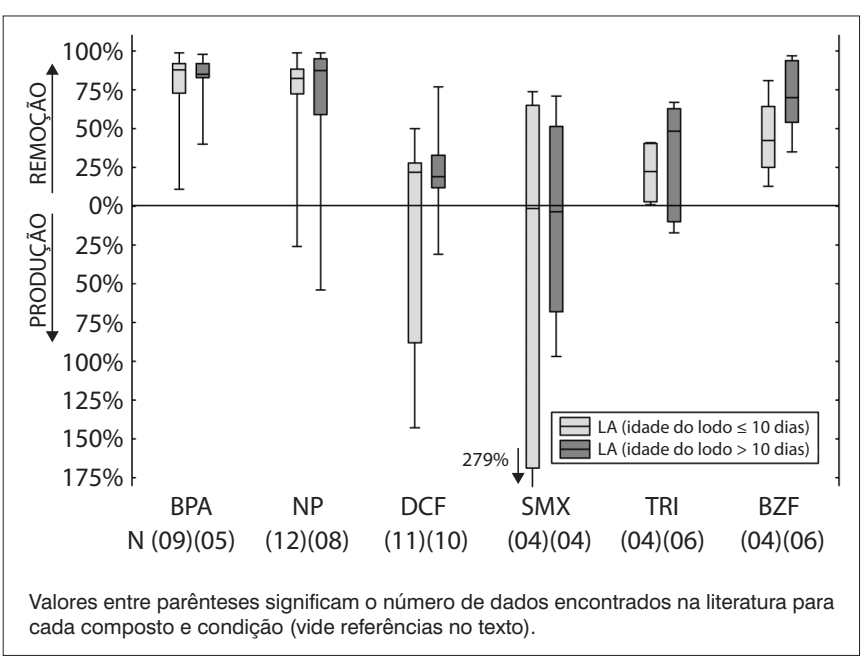

Figura 2 - Eficiências de remoção de alguns fármacos e DE em sistemas de lodos ativados (LA) e influência do tempo de retenção dos sólidos nos sistemas (idade do lodo).
CLARA et al., 2005b), além de ser o maior valor admitido para que um sistema de lodos ativados seja definido como convencional (VON SPERLING, 2002). O número de dados (N) encontrados na literatura para cada composto nas duas condições operacionais também está apresentado na Figura 2.

Da Figura 2 depreende-se que os fármacos DCF e SMX passam praticamente incólumes pelo sistema de lodos ativados, ao passo que outros, como TRI e BZF, apresentam remoção parcial. Finalmente, existem aqueles que apresentam eficiência de remoção mais elevada nesse tipo de tratamento, como é o caso de BPA e NP.

Alguns artigos revisados relatam inclusive o aumento da concentração de alguns fármacos (ex.: DCF, SMX e TRI) ao longo do sistema de tratamento, o que é , em geral, atribuído ao fenômeno de hidrólise de conjugados originalmente presentes no esgoto bruto ou à degradação incompleta de precursores. A conversão de compostos químicos em conjugados (ex.: glicuronídeos, sulfatos) é um método utilizado pelos organismos para auxiliar na solubilização e excreção de substâncias pouco solúveis em água, como é o caso de grande parte dos fármacos e dos DE. No caso do NP, a produção observada em alguns sistemas de tratamento (ex.: reatores anaeróbios) é atribuída à degradação de compostos precursores, a exemplo dos alquilfenóis polietoxilados (APEO), presentes nas formulações de produtos de limpeza (GUENTHER et al., 2006).

A Figura 2 mostra ainda que os xenoestrogênios (BPA e NP) são relativamente bem removidos (medianas $>80 \%$ ), independente da idade do lodo no sistema de lodos ativados. Devido aos elevados valores de log de $\mathrm{K}_{\mathrm{ow}}$ do BPA e do NP (Tabela 4), a sorção é apontada como o principal mecanismo de remoção dessas substâncias em sistemas de tratamento de esgoto por lodos ativados (CIRJA et al., 2008).

No caso do DCF, fármaco com baixo $\log \mathrm{K}_{\mathrm{d}}$ (Tabela 3) e $\mathrm{K}_{\mathrm{bio}}<0,1$ L.g ${ }_{s s}{ }^{-1} \cdot d^{-1}$ (JOSS et al., 2006), espera-se baixa remoção por sorção e baixa biodegradabilidade, independentemente da idade do lodo (SUÁREZ et al., 2008). Tal premissa foi confirmada pelas baixas eficiências de remoção encontradas na literatura (medianas<20\%), aparentemente independentes da idade do lodo (Figura 2). Porém, é importante observar a maior variabilidade dos dados e a maior quantidade de resultados de produção do DCF nos sistemas de lodos ativados com idades do lodo menores ou iguais a 10 dias, o que de certa forma demonstra pequena dependência da remoção do DCF desse parâmetro operacional. Possivelmente, os sistemas com idades do lodo maiores do que 10 dias são mais eficientes na remoção do DCF do que aqueles com idades do lodo menores, pois além de removerem parte do DCF presente no esgoto sanitário, levam à degradação de praticamente todo o fármaco formado a partir da hidrólise dos conjugados — infelizmente há pouquíssimos dados de produção do DCF em ETE com idades do lodo superiores a 10 dias para comparação. 
Ainda na Figura 2, percebe-se que, em termos medianos, os sistemas de lodos ativados, mesmo aqueles operados com idades de lodo superiores a 10 dias, não removem SMX. Tal como no caso do DCF, observa-se uma menor variabilidade dos dados e uma menor produção deste antibiótico nos sistemas operados com maior idade do lodo. Segundo Suárez et al. (2008), fármacos e DE com baixos valores de $\mathrm{K}_{\text {bio }}$ e $\mathrm{K}_{\mathrm{d}}$, como é o caso do SMX, não são retidos nas unidades de tratamento nem biodegradados, independente da idade do lodo, o que de certa forma é respaldado pelos dados da literatura aqui compilados.

Com relação à TRI e ao BZF, os dados da literatura (Figura 2) mostram que tais compostos são moderadamente transformados nos sistemas de lodos ativados, com valores medianos de remoção que variam de 20 a $75 \%$ e que tendem a ser dependentes da idade do lodo. Esse efeito é observado provavelmente porque os compostos apresentam valores de $\mathrm{K}_{\text {bio }}$ na faixa baixa/intermediária, além de moderada tendência para a sorção (Tabelas 3 e 4). Portanto, maior idade do lodo permite também maior tempo para a retenção dos compostos no sistema de tratamento e sua subsequente biodegradação.

\section{Outros sistemas de tratamento}

Estudos sobre remoção de fármacos e DE em outros sistemas de tratamento que não os lodos ativados são muito menos frequentes, talvez pelo fato de serem menos utilizados nos países (EUA, Canadá, Coreia do Sul, China, Japão e países europeus) onde são realizadas a maior parte das pesquisas no tema (CLARA et al., 2005a,b; GULKOWSKA et al., 2008; KIM et al., 2007; LI \& ZHANG, 2010; MIĖGE et al., 2008; SIPMA et al., 2009; TERNES et al.,1999). Verifica-se que há, na literatura, pouquíssimos dados de remoção de fármacos e DE em ETE compostas por filtros biológicos percoladores (FBP) e reatores anaeróbios (ex.: reatores UASB, reatores EGSB Expanded Granular Sludge Bed). Já para os sistemas naturais, a exemplo das lagoas de estabilização e dos alagados construídos (wetlands), são encontrados mais estudos, se bem que esparsos.

A Tabela 6 sumariza a remoção de alguns fármacos e DE em FBP, lagoas de estabilização, alagados construídos e sistemas anaeróbios de tratamento de esgoto. Observa-se ampla variação nas eficiências de remoção relatadas na literatura para todos os compostos e processos de tratamento, mas, de maneira geral, há certa convergência para baixas eficiências de remoção de fármacos e DE com menores tendências de adsorção nos FBP e eficiências intermediárias ou elevadas para praticamente todos os compostos em sistemas naturais de tratamento (lagoas de estabilização e alagados construídos). É possível que os maiores valores de TDH e a maior exposição à luz solar verificadas nos processos naturais favoreçam os mecanismos de biodegradação e fotodegradação desses microcontaminantes. No caso dos FBP, que são sistemas de tratamento compactos com baixos valores de TDH, são observadas maiores eficiências de remoção apenas para aqueles compostos que apresentam maior tendência para a sorção e que têm valores de $\mathrm{K}_{\text {bio }}$ mais elevados. Possivelmente, o tempo de contato dos contaminantes sorvidos na biomassa é um fator determinante para a sua remoção em tais sistemas.

Embora o potencial dos processos anaeróbios para a remoção de microcontaminantes já tenha sido estudado em escala de bancada por diferentes grupos de pesquisa (EJLERTSSON et al., 1999; JÜRGENS et al., 2002; LEE \& LIU, 2002; JOSS et al., 2004), há pouquíssimos estudos que avaliaram o comportamento de alguns fármacos e DE em reatores anaeróbios do tipo UASB (QUEIROZ et al., 2012; REYES et al., 2010; GRAAFF et al., 2011) e ainda menos pesquisas em lagoas anaeróbias (SERVOS et al., 2005). Em pesquisa recente, Queiroz et al. (2012) avaliaram o comportamento de diversos fármacos e DE em reatores UASB operados sob diferentes TDH e concluíram que: (i) os reatores UASB foram ineficientes na remoção do BPA e levaram a um aumento da concentração do NP no efluente; (ii) o aumento das concentrações do NP provavelmente ocorreu devido à degradação anaeróbia dos APEO, sendo que essa degradação pareceu ser diretamente proporcional ao TDH; (iii) os reatores anaeróbios também foram ineficientes na remoção do DCF e levaram a uma remoção parcial do BZF, sendo que para o SMX, não houve uma relação direta entre o TDH e as eficiências de remoção; (iv) para a TRI, o tempo de retenção dos sólidos pareceu desempenhar papel importante em sua remoção, embora tal antibiótico tenha sido apenas parcialmente removido nos reatores UASB.

Tabela 6 - Faixas de eficiências médias relatadas em estudos de remoção de fármacos e desreguladores endócrinos em filtros biológicos percoladores, lagoas de estabilização, alagados construídos e reatores UASB.

\begin{tabular}{|c|c|c|c|c|c|c|c|c|c|}
\hline \multirow{2}{*}{ Sistema de tratamento } & \multicolumn{9}{|c|}{ Eficiências de remoção (mín./máx. \%)a } \\
\hline & E1 & E2 & EE2 & BPA & NP & DCF & SMX & TRI & BZF \\
\hline Filtros biológicos percoladores ${ }^{1-5}$ & $50 / 67$ & $81 / 92$ & 64 & $65 / 91$ & $54 / 83$ & $-85 / 54$ & $-21 / 98$ & $-39 / 40$ & $-59 / 86$ \\
\hline Lagoas de estabilização (exceto anaeróbias) ${ }^{1,6-10}$ & $1 / 100$ & $32 / 98$ & $25 / 99$ & $20 / 100$ & $-9 / 85$ & 70 & $28 / 78$ & $66 / 94$ & 42 \\
\hline Alagados construídos (wetlands) ${ }^{1,10-15}$ & 1 & I & l & 1 & l & $0 / 96$ & $15 / 66$ & $36 / 56$ & 1 \\
\hline Lagoas anaeróbias ${ }^{8}$ & 96 & 98 & 1 & 1 & 1 & 1 & 1 & 1 & 1 \\
\hline Reatores UASB ${ }^{16-18}$ & 1 & 1 & 1 & $-87 /-5$ & $-214 /-194$ & $-41 / 22$ & $-37 / 60$ & $45 / 100$ & $-59 / 40$ \\
\hline
\end{tabular}

(1) Brandt (2012); (2) Ternes et al., 1999; (3) Kasprzyk-Hordern, Dinsdale e Guwy 2009; (4) Spengler et al., 2001; (5) Jiang et al., 2005; (6) Froehner et al., 2011; (7) Ying, Kookana e Kumar 2008; (8) Servos et al., 2005; (9) Karthikeyan et al., 2006; (10) Conkle, White e Metcalfe 2008; (11) Matamoros et al., 2006; (12) Matamoros et al., 2008; (13) Matamoros et al., 2009; (14) Verlicchi et al., 2010; (15) Galletti et al., 2010; (16) Queiroz et al., 2012; (17) Reyes et al., 2010; (18) Graaff et al., 2011.

as eficiências negativas são devido à produção do contaminante no sistema de tratamento, provavelmente devido ao fenômeno de desconjugação ou degradação de compostos precursores 


\section{Conclusão}

A ocorrência de fármacos e desreguladores endócrinos em cursos d'água constitui ameaça potencial aos organismos aquáticos e à saúde pública. A literatura mostra que os esgotos domésticos representam uma importante rota de contaminação dos ambientes aquáticos, e que apenas um pequeno grupo de tais compostos é removido satisfatoriamente nos sistemas de tratamento de esgoto ditos convencionais, que empregam processos biológicos.

De forma geral, a remoção nas unidades da ETE é fortemente dependente das propriedades físico-químicas das moléculas dos fármacos e DE, das configurações dos sistemas de tratamento e das condições operacionais aplicadas às unidades de tratamento. Os estrogênios naturais e os xenoestrogênios são os DE mais facilmente removidos (eficiências de remoção $>80 \%$ ), possivelmente devido a sua grande tendência para sorção (valores elevados de $\log \mathrm{K}_{\mathrm{ow}}$ e $\log \mathrm{K}_{\mathrm{d}}$ ) e à favorável cinética de biodegradação (elevados valores de $\mathrm{K}_{\text {bio }}$ ), como é o caso dos estrogênios naturais. Para os fármacos mais frequentemente detectados no esgoto, é de se esperar eficiências de remoção que variam de intermediária (20 a 80\%) a baixa ( $<20 \%$ ), a depender da capacidade de sorção dos microcontaminantes e dos parâmetros operacionais aplicados aos sistemas de tratamento, notadamente a idade do lodo e o TDH. Para fármacos ácidos ou básicos, a sorção depende do grau de ionização da molécula e é altamente afetada pelo pH de operação do sistema de tratamento.

Nos casos em que o processo de adsorção é um importante mecanismo de remoção dos fármacos e dos DE, deve-se atentar para as concentrações dos compostos no lodo e nos sólidos suspensos do efluente, tendo em vista que a transferência de fases não elimina o problema ambiental ou de saúde. Dessa forma, devem-se avaliar os riscos associados à disposição final ou ao reúso do lodo contendo tais microcontaminantes, assunto ainda incipiente na literatura, principalmente no nosso país.

Com base no exposto, pode-se concluir que a obtenção de elevadas eficiências (>90\%) de remoção de fármacos e DE em sistemas de tratamento de esgoto passa pela adoção de sistemas de tratamento terciário, que empregam processos físico-químicos, tais como a adsorção em carvão ativado e a oxidação química (convencional e avançada). A adoção de tais sistemas complementares de tratamento envolve, entretanto, análise de custo-benefício, o que depende ainda de avaliação criteriosa dos riscos causados por tais contaminantes no ambiente, principalmente para a saúde humana, tema ainda controverso na literatura.

\section{Agradecimentos}

A CAPES, CNPq, Finep e Fapemig, agências brasileiras que têm financiado projetos dos autores nessa área de pesquisa.

\section{Referências}

AHERNE, G.W.; ENGLISH, J.; MARKS, V. (1985) The role of immunoassay in the analysis of microcontaminants in water samples. Ecotoxicology and Environmental Safety, v. 9, p. 79-83.

ANDERSEN, H.R.; HANSEN, M.; KJØLHOLT, J.; STUER-LAURIDSEN, F.; TERNES, T.; HALLING-SØRENSEN, B. (2005) Assessment of the importance of sorption for steroid estrogens removal during activated sludge treatment. Chemosphere, v. 61, p. 139-146.

ANDREOZZI, R.; RAFFAELE, M.; NICKLAS, P. (2003) Pharmaceuticals in STP effluents and their solar photodegradation in aquatic environment. Chemosphere, v. 50, p. 1319-1330.

ASH, R.J. \& IVERSON, J.L. Antibiotic and disinfectant resistant bacteria in rivers of the United States. Proceedings of the $4^{\text {th }}$ International Conference on Pharmaceuticals and Endocrine Disrupting Chemicals in Water. Minneapolis, EUA, 2004.

BATT, A.L.; KIM, S.; AGA, D.S. (2006) Enhanced Biodegradation of Iopromide and Trimethoprim in Nitrifying Activated Sludge. Environmetal Science \& Technology, v. 40, p. 7367-7373.

BERNHARD, M.; MÜLLER, J.; THOMAS, P.K. (2006) Biodegradation of persistent polar pollutants in wastewater: Comparison of an optimized lab-scale membrane bioreactor and activated sludge treatment. Water Research, v. 40, p. 3419-3428.

BILA, D.M. \& DEZZOTTI, M. (2007) Desreguladores endócrinos no meio ambiente: efeitos e consequências. Química Nova, v. 30, p. 651-666.
BURGESS, R.M.; PELLETIER, M.C.; GUNDERSEN, J.L.; PERRON, M.M.; RYBA, S.A. (2005) Effects of different forms of organic carbon on the partitioning and bioavailability of nonylphenol. Environmental Toxicology \& Chemistry, v. 24, n. 7, p. 1609-1617.

BRANDT, E.M.F. (2012) Avaliação da remoção de fármacos e desreguladores endócrinos em sistemas simplificados de tratamento de esgoto (reatores UASB seguidos de pós-tratamento). 128 p. Dissertação (Mestrado em Saneamento, Meio Ambiente e Recursos Hídricos) Universidade Federal de Minas Gerais, Belo Horizonte (MG), Brasil.

BUSER, H.; POIGER, T.; MÜLLER, M.D. (1998) Pharmaceutical Drug Diclofenac in Surface Waters: Rapid Photodegradation in a Lake. Environmental Science \&Technology, v. 32, n. 22.

CARBALLA, M.; OMIL, F.; LEMA, J. M.; LLOMPART, M.; GARCÍAJARES, C.; RODRÍGUEZ, I.; GÓMEZ, M.; TERNES, T. (2004) Behavior of pharmaceuticals, cosmetics and hormones in a sewage treatment plant. Water Research, v. 38, p. 2918-2926.

CARBALLA, M.; OMIL, F.; LEMA, J.M. (2007) Calculation methods to perform mass balances of micropollutants in sewage treatment plants. Application to pharmaceutical and personal care products (PPCPS). Environmental Science \& Technology, v. 41, p. 884-890.

CARBALLA, M.; FINK, G.; OMIL, F.; LEMA, J.M.; TERNES, T. (2008) Determination of the solid-water distribution coefficient $(K d)$ for pharmaceuticals, estrogens and musk fragrances in digested sludge. Water Research, v. 42, p. 287-295. 
CARLSEN, E.; GIWERCMAN, A.; KEIDING, N.; SKAKKEBAEK, N.E. (1992) Evidence for decreasing quality of semen during past 50 years. British Medical Journal, v. 305, n. 6854, p. 609-613.

CHOUBERT, J.M.; RUEL, S.M.; ESPERANZA, M.; BUDZINSKI, H.; MIĖGE, C.; LAGARRIGUE, C.; COQUERY, M. (2011) Limiting the emissions of micro-pollutants: what efficiency can we expect from wastewater treatment plants? Water Science \& Technology, v. 63, p. 57-65.

CHOWDHURY, R.R.; CHARPENTIER, P.A.; RAY, M.B. (2010) Photodegradation of estrone in solar irradiation. Industrial \& Engineering Chemistry Research, v. 49, n.15, p.6923-6930.

CHOWDHURY, R.R.; CHARPENTIER, P.A.; RAY, M.B. (2011) Photodegradation of $17 \beta$-estradiol in aquatic solution under solar irradiation: Kinetics and influencing water parameters. Journal of Photochemistry and Photobiology A: Chemistry, v. 219, p.67-75.

CIRJA, M.; IVASHECHKIN, P.; SCHÄFFER, A.; CORVINI, P.F.X. (2008) Factors affecting the removal of organic micropollutants from wastewater in conventional treatment plants (CTP) and membrane bioreactors (MBR), review article. Reviews in Environmental Science and Biotechnology, v. 7, p. 61-78.

CLARA, M.; STRENN, B.; AUSSERLEITNER, M.; KREUZINGER, N. (2004) Comparison of the behavior of selected micropollutants in a membrane bioreactor and a conventional wastewater treatment plant. Water Science \& Technology, v. 50, n. 5, p. 29-36

CLARA, M.; STRENN, B.; GANS, O.; MARTINEZ, E.; KREUZINGER, N.; KROISS, H. (2005a) Removal of selected pharmaceuticals, fragrances and endocrine disrupting compounds in a membrane bioreactor and conventional wastewater treatment plants. Water Research, v. 39, p. 4797-4807.

CLARA, M.; KREUZINGER, N.; STRENN, B.; GANS, O.; KROISS, H. (2005b) The solids retention time - a suitable design parameter to evaluate the capacity of wastewater treatment plants to remove micropollutants. Water Research, v.39, p. 97-106.

CONKLE, J.; WHITE, J.R.; METCALFE, C.D. (2008) Reduction of pharmaceutically active compounds by a lagoon wetland wastewater treatment system in Southeast Louisiana. Chemosphere, v. 73, n. 11, p. 1.741-1.748.

EJLERTSSON, J.; NILSSON, M-L.; KYLIN, H.; BERGMAN, A.; KARLSON, L.; ÖQUIST, M.; SVENSSON, B.H. (1999) Anaerobic degradation of nonylphenol mono-and diethoxylates in digester sludge, landfilled municipal solid waste, and landfilled sludge. Environmental Science \& Technology, v. 33, p. 301-306

FROEHNER, S.; PICCIONI, W.; MACHADO, K.S.; AISSE, M.M. (2011) Removal Capacity of Caffeine, Hormones, and Bisphenol by Aerobic and Anaerobic Sewage Treatment. Water, Air \& Soil Pollution, v. 216, p. 463-471.

GALASSI, S.; VALESCCHI, S.; TARTARI, G.A. (1997) The distribution of PCB's and chlorinated pesticides in two connected Himalayan lakes. Water, Air, \& Soil Pollution, v. 99, n. 1-4, p. 717-725.
GALLETTI, A.; AL AUKIDI, M.; VERLICCHI, P.; PETROVIC, M.; BARCELÓ, D. (2010) Pharmaceuticals removal in a H-SSF treating a secondary domestic wastewater - an experimental investigation. Proceedings of the International Conference on Wetland Systems for Water Pollution Control, 12 $2^{\text {th }}$, Venice: IWA, 2010

GHISELLI, G. (2006) Avaliação da Qualidade das Águas Destinadas ao Abastecimento Público na Região de Campinas: Ocorrência e Determinação dos Interferentes Endócrinos (IE) e Produtos Farmacêuticos e de Higiene Pessoas (PFHP). 190 p. Tese (Doutorado em Ciências) Universidade Estadual de Campinas, Campinas, 2006.

GHISELLI, G. \& JARDIM, W.F. (2007) Interferentes endócrinos no ambiente. Química Nova, v. 30, p. 695-706.

GÖBEL, A.; THOMSEN, A.; McARDELL, C.S.; JOSS, A.; GIGER, W. (2005) Occurrence and sorption behavior of sulfonamides, macrolides and trimethoprim in conventional activated sludge treatment. Environmental Science \& Technology, v. 39, n. 11, p. 3981-3989.

GÖBEL, A.; McARDELL, C.S.; JOSS, A.; SIEGRIST, H.; GIGER, W. (2007) Fate of sulfonamides, macrolides, and trimethoprim in different wastewater treatment technologies. Science of the Total Environment, v. 372 , p. 361-371.

GOMES, R.L.; SCRIMSHAW, M.D.; LESTER, J.N. (2009) Fate of Conjugated Natural and Synthetic Steroid Estrogens in Crude Sewage and Activated Sludge Batch Studies. Environmental Science and Technology, v. 43, p. 3612-3618

GRAAFF, M.S.; VIENO, N.M.; KUJAWA-ROELEVELD, K.; ZEEMAN G.; TEMMINK, H.; BUISMAN, C.J.N. (2011) Fate of hormones and pharmaceuticals during combined anaerobic treatment and nitrogen removal by partial nitritation anammox in vacuum collected black water. Water Research, v. 45, p. 375-383.

GUENTHER, K.; KLEIST, E.; THIELE, B. (2006) Estrogen-active nonylphenols from an isomer-specific viewpoint: a systematic numbering system and future trends. Analytical and Bioanalytical Chemistry, v. 384, p. 542-546.

GUILLETTE, L.J. Jr.; PICKFORD, D.B.; CRAIN, D.A.; ROONEY, A.A.; PERCIVAL, H.F. (1996) Reduction in penis size and plasma testosterone concentrations in juvenile alligators living in a contaminated environment. General and Comparative Endocrinology, v. 101, n. 1, p. 32-42.

GULKOWSKA, A.; LEUNG, H.W.; SO, M.K.; TANIYASU, S.; YAMASHITA, N.; YEUNG, L.W.Y.; RICHARDSON, B.J.; LEI, A.P.; GIESY, J.P.; LAM, P.K.S (2008) Removal of antibiotics from wastewater by sewage treatment facilities in Hong Kong and Shenzhen, China. Water Research, v. 42, p. 395-403.

GURR, C.J. \& REINHARD, M. (2006) Harnessing natural attenuation of pharmaceuticals and hormones in rivers. Environmental Science \& Technology, v. 40, n. 9, p. 2.872-2.876.

HALLING-SØRENSEN, B.; NIELSEN, S.N.; LANZKY, P.F.; INGERSLEV F.; LUTZHEFT, H.C.H.; JØRGENSEN S.E. (1998) Occurrence, Fate and Effects of Pharmaceutical Substances in the Environment - A review. Chemosphere, v. 36, n. 2, p. 357-393 
HIGNITE, C.; AZARNOFF, D.L. (1977) Drugs and drug metabolites as environmental contaminants: Chlorophenoxyisobutyrate and salicylic acid in sewage water effluent. Life Sciences, v. 20, n. 2, p. 337-341.

IWA, International Water Association. (2010) Treatment of Micropollutants in Water and Wastewater. VIRKUTYTE, J.; VARMA, R.S.; JEGATHEESAN, V. (Eds.). London. 483p.

JANEX-HABIB, M.; HUYARD, A.; ESPERANZA, M.; BRUCHET, A. (2009) Reduction of endocrine disruptor emissions in the environment: The benefit of wastewater treatment. Water Research, v. 43, p. 1565-1576.

JELIC, A.; GROS, M.; GINEBREDA, A.; CESPEDES-SÁNCHEZ, R.; VENTURA, F.; PETROVIC, M.; BARCELO, D. (2011) Occurrence, partition and removal of pharmaceuticals in sewage water and sludge during wastewater treatment. Water Research, v. 45, p. 1165-1176.

JIANG, J.Q.; YIN, Q.; ZHOU, J.L.; PEARCE, P. (2005) Occurrence and treatment trials of endocrine disrupting chemicals (EDCs) in wastewaters. Chemosphere, v. 61, p. 544-550

JOBLING, S.; NOLAN, M.; TYLER, C.R.; BRIGHTY, G.; SUMPTER, J. P. (1998) Widespread Sexual Disruption in Wild Fish. Environmental Science \& Technology, v. 32, p. 2498-2506.

JOSS, A.; ANDERSEN, H.; TERnES, T.; RICHLE, P.R.; SIEGRIST, H. (2004) Removal of estrogens in municipal wastewater treatment under aerobic and anaerobic conditions: consequences for plant optimization. Environmental Science \& Technology, v. 38, n. 11, p. 3047-3055

JOSS, A.; KELLER, E.; ALDER, A.C.; GÖBEL, A.; MCARDELL, C.S.; TERNES, T.; SIEGRIST, H. (2005) Removal of pharmaceuticals and fragrances in biological wastewater treatment. Water Research, v. 39, p. 3139-3152

JOSS, A.; ZABCZYNSKI, S.; GÖBEL, A.; HOFFMANN, B.; LÖFFLER, D.; McARDELL, C.S.; TERNES, T.A.; THOMSEN, A.; SIEGRIST, H. (2006) Biological degradation of pharmaceuticals in municipal wastewater treatment: Proposing a classification scheme. Water Research, v. 40, p. 1686-1696

JÜRGENS, M.D.; HOLTHAUS, K.I.E.; JOHNSON, A.C.; SMITH, J.J.L.; HETHERIDGE, M.; WILLIAMS, R.J. (2002) The potential for estradiol and ethinylestradiol degradation in English rivers. Environmental Toxicology \& Chemistry, v. 21, n. 3, p. 480-488.

KARTHIKEYAN, K.G. \& MEYER, M.T. (2006) Occurrence of antibiotics in wastewater treatment facilities in Wisconsin, USA. Science of the Total Environment, v. 361, p. 196-207.

KASHIWADA, S.; ISHIKAWA, H.; MIYAMOTO, N.; OHNISHI, Y.; MAGARA, Y. (2002) Fish test for endocrine-disruption and estimation of water quality of Japanese rivers. Water Research, v. 36, p. 2161-2166.

KASPRZYK-HORDERN, B.; DINSDALE, R.M.; GUWY, A.J. (2009) The removal of pharmaceuticals, personal care products, endocrine disruptors and illicit drugs during wastewater treatment and its impact on the quality of receiving waters. Water Research, v. 43, p. 363-380.

KIM, S. \& AGA, D.S. (2007) Potential ecological and human health impacts of antibiotics and antibiotic-resistant bacteria from wastewater treatment plants. Journal of Toxicology and Environmental Health, Part B, v. 10, n. 8, p. $559-573$.

KIM, S.D.; CHO, J.; KIM, I.S.; VANDERFORD, B. J.; SNYDER, S.A. (2007) Occurrence and removal of pharmaceuticals and endocrine disruptors in South Korean surface, drinking, and waste waters. Water Research, v. 41, p. 1013-1021.

KÜMMERER, K. (2010) Pharmaceuticals in the Environment. The Annual Review of Environment and Resources, v. 35, p. 57-75.

LEE, H.B. \& LIU, D. (2002) Degradation of $17 \beta$-estradiol and its metabolites by sewage bacteria. Water, Air \& Soil Pollution, v. 134, n. 1-4, p. 353-368.

LEECH, D.M.; SNYDER, M.T.; WETZEL, R.G. (2009) Natural organic matter and sunlight accelerate the degradation of $17 \beta$-estradiol in water. Science of the Total Environment, v. 407, p. 2087-2092.

LI, B. \& ZHANG, T. (2010) Biodegradation and adsorption of antibiotics in the activated sludge process. Environmental Science and Technology, v. 44 , p. $3468-3473$

LINDBERG, R.H.; WENNBERG, P.; JOHANSSON, M.I.; TYSKLIND, M.; ANDERSSON, B.A.V. (2005) Screening of human antibiotic substances and determination of weekly mass flows in five sewage treatment plants in Sweden. Environmental Science and Technology, v. 39, n. 5, p. 3421-3429

LISHMAN, L.; SMYTH, S.A.; SARAFIN, K.; KLEYWEGT, S.; TOITO, J.; PEART, T.; LEE, B.; SERVOS, M.; BELAND, M.; SETO, P. (2006) Occurrence and reductions of pharmaceuticals and personal care products and estrogens by municipal wastewater treatment plants in Ontario, Canada. Science of the Total Environment, v. 367, p. 544-558.

LOCATELLI, M.A.F.; SODRÉ, F.F.; JARDIM, W.F. (2011) Determination of Antibiotics in Brazilian Surface Water Using Liquid ChromatographyElectrospray Tandem Mass Spectrometry. Archives of Environmental Contamination and Toxicology, v. 60, p. 385-393.

MAKI, H.; FUJITA, M.; FUJIWARA, Y. (1996) Identification of final biodegradation product of nonylphenol ethoxylate (NPE) by river microbial consortia. Bulletin of Environmental Contamination and Toxicology, v. 57, p. 881-887.

MANZANO, M.A.; PERALES, J.A.; SALES, D.; QUIROGA, J.M. (1999) The effect of temperature on the biodegradation of a nonylphenol polyethoxylate in river water. Water Research, v. 33, p. 2593-2600.

MATAMOROS, V. \& BAYONA, J.M. (2006) Elimination of pharmaceuticals and personal care products in subsurface flow constructed wetlands. Environmental Science \& Technology, v. 40, p. 5811-5816.

MATAMOROS, V.; GARCÍA, J.; BAYONA, J.M. (2008) Organic micropollutant removal in a full scale surface flow constructed wetland fed with secondary effluent. Water Research, v. 42, p. 653-660.

MATAMOROS, V.; ARIAS, C.; BRIX, H.; BAYONA, J.M. (2009) Preliminary screening of small-scale domestic wastewater treatment systems for removal of pharmaceutical and personal care products. Water Research, v. 43, p. $55-62$. 
McADAM, E.J.; BAGNALL, J.P.; SOARES, A.; KOH, Y.K.K.; CHIU, T. Y.; SCRIMSHAW, M.D.; LESTER, J.N.; CARTMELL, E. (2011) Fate of Alkylphenolic Compounds during Activated Sludge Treatment: Impact of Loading and Organic Composition, Environmental Science \& Technology, v. 45, p. 248-254.

MES, T.; ZEEMAN, G.; LETTINGA, G. (2005) Occurrence and fate of estrone, $17 \beta$ - estradiol and $17 \alpha$-ethynylestradiol in STPs for domestic wastewater. Reviews in Environmental Science and Bio/Technology, v. 4, p. 275-311.

MIÈGE, C.; CHOUBERT, J.M.; RIBEIRO, L.; EUSĖBE, M.; COQUERY, M. (2008) Removal efficiency of pharmaceuticals and personal care products with varying wastewater treatment processes and operating conditions - conception of a database and first results. Water Science \& Technology, v. 57, n. 1, p. 49-56.

MIRANDA, C.D.; CASTILLO, G. (1998) Resistance to antibiotic and heavy metals of motile aeromonads from Chilean freshwater. Science of the Total Environment, v. 224, n. 1-3, p. 167-176.

MONTAGNER, C.C. (2007) Ocorrência de interferentes endócrinos e produtos farmacêuticos nas águas superficiais da bacia do rio Atibaia. 126 p. Dissertação (Mestrado em Química), Universidade Estadual de Campinas, Campinas (SP), Brasil.

MONTAGNER, C.C. \& JARDIM, W.F. (2011) Spatial and Seasonal Variations of Pharmaceuticals and Endocrine Disruptors in the Atibaia River, São Paulo State (Brazil). Journal of the Brazilian Chemical Society v. 22, p. 1452-1462.

MOREIRA, D.S.; AQUINO, S. F.; AFONSO, R. J. C. F.; SANTOS, E. P. P. C.; PÁDUA, V.L. (2009) Occurrence of endocrine disrupting compounds in water sources of Belo Horizonte Metropolitan Area, Brazil. Environmental Technology, v. 30, n. 10, p. 1041-1049.

MOREIRA, M.A.; AQUINO, S.F.; COUTRIM, M.X.; SILVA, J.C.C.; AFONSO, R.J.C.F. (2011) Determination of endocrine-disrupting compounds in waters from Rio das Velhas, Brazil, by liquid chromatography/high resolution mass spectrometry (ESILC-IT-TOF/MS). Environmental Technology, v. 32, n. (11-12), p. 1409-1417.

NAKADA, N.; TANISHIMA, T.; SHINOHARA, H.; KIRI, K.; TAKADA, $H$. (2006) Pharmaceutical chemicals and endocrine disrupters in municipal wastewater in Tokyo and their removal during activated sludge treatment. Water Research, v. 40, p. 3297-3303.

NASSIF M.C., CIMAROSTI H.I., ZAMIN L.L., SALBEGO C.G. (2005) Estrógeno versus isquemia cerebral: hormônio feminino como agente neuroprodutor. Infarma, v. 17, p. 57-65.

PEDROUZO, M.; BORRULL, F.; POCURULL, E.; MARCÉ, R.M. (2011) Presence of pharmaceuticals and hormones in waters from sewage treatment plants. Water, Air, \& Soil Pollution, v. 217, n. 1-4, p. 267-281.

PESSOA, G.P.; SOUZA, N.C.; ALVES, J.A.C.; NASCIMENTO, R.F.; SANTOS, A.B. (2011) Análise de remoção de interferentes endócrinos em estações de tratamento de esgotos sanitários. In: $26^{\circ}$ Congresso
Brasileiro de Engenharia Sanitária e Ambiental, Anais... Porto Alegre: Associação Brasileira de Engenharia Sanitária e Ambiental, 2011

POMATI, F.; ORLANDI, C.; CLERICI, M.; LUCIANI, F.; ZUCCATO, E. (2008) Effects and interactions in environmentally relevant mixture of pharmaceuticals. Toxicological Sciences, v. 102, p. 129-137.

POTHITOU, P. \& VOUTSA, D. (2008) Endocrine disrupting compounds in municipal and industrial wastewater treatment plants in Northern Greece. Chemosphere, v. 73, p. 1716-1723.

PURDOM, C.E.; HARDIMAN, P.A.; BYE, V.J.; ENO, N.C.; TYLER, C. R.; SUMPTER, J. P. (1994) Estrogenic effects of effluent from sewage treatment works. Chemistry in Ecology, v. 8, p. 275-285.

QUEIROZ, F.B.; BRANDT, E.M.F.; AQUINO, S.F.; CHERNICHARO, C.A.L.; AFONSO, R.J.C.F. (2012) Occurrence of pharmaceuticals and endocrine disruptors in raw sewage and their behavior in UASB reactors operated at different hydraulic retention times. Water Science \& Technology, v. 6612, p. 2562-2569

QUINN, B.; GAGNÉ, F.; BLAISE, C. (2009) Evaluation of the acute, chronic and teratogenic effects of a mixture of eleven pharmaceuticals on the cnidarian, Hydra attenuate. Science of the Total Environment, v. 407, p. $1072-1079$.

RADJENOVIC, J.; PETROVIC, M.; BARCELÓ, D. (2007) Analysis of pharmaceuticals in wastewater and removal using a membrane bioreactor. Analytical and Bioanalytical Chemistry, v. 387, p. 1365-1377.

RADJENOVIC, J.; PETROVIC, M.; BARCELÓ, D. (2009) Fate and distribution of pharmaceuticals in wastewater and sewage sludge of the conventional activated sludge (CAS) and advanced membrane bioreactor (MBR) treatment. Water Research, v. 43, p.831-841.

REYES, C.; MATAMOROS, V.; CRUJEIRAS, B.; RUIZ, I.; SOTO, M.; BAYONA, J. M. (2010) Evaluation of an aerobic digester (UASB) followed by a hybrid constructed wetland for the removal of PPCPs in urban wastewater: A pilot study. In: $12^{\text {th }}$ International Conference on Wetland Systems for Water Pollution Control, Proceedings..., Venice: International Water Association , 2010.

RIBEIRO, A.R.; CARVALHO, M.F.; AFONSO, C.M.M.; TIRITAN, M.E.; CASTRO, P.M.L. (2010) Microbial degradation of 17-estradiol and 17-ethynylestradiol followed by a validated HPLC-DAD method. Journal of Environmental Science and Health - Part B - Pesticides, food contaminants and agricultural wastes, v. 5, n. 4, p. 265-273.

ROGERS, H.R. (1996) Sources, behavior and fate of organic contaminants during sewage treatment and in sewage sludges. Science of the Total Environment, v. 185, p. 3-26.

SAIANO, $H_{\text {.; }}$ JAMAGATA, $H_{\text {. }}$; NAKAJIMA, $H_{\text {. }}$; SHIGEMURA, $H_{\text {.; }}$ SUZUKI, Y. (2004) Removal of endocrine disrupting chemicals in wastewater by SRT control. Journal of Japan Society on Water Environment, v. 27, p. 61-68.

SANTOS, L.H.M.L.M.; ARAÚJO, A.N.; FACHINI, A.; PENA, A.; DELERUEMATOS, C.; MONTENEGRO, M.C.B.S.M. (2010) Ecotoxicological 
aspects related to the presence of pharmaceuticals in the aquatic environment. Journal of Hazardous Materials, v. 175, p. 45-95.

SERVOS, M.R.; BENNIE, D.T.; BURNISON, B.K.; JURKOVIC, A.; McINNIS, R.; NEHELI, T.; SCHNELL, A.; SETO, P.; SMYTH, S.A.; TERNES, T.A. (2005) Distribution of estrogens, 17b-estradiol and estrone, in Canadian municipal wastewater treatment plants. Science of The Total Environment, v. 336, n. 1-3, p. 155-170

SIPMA, J.; OSUNA, B.; COLLADO, N.; MONCLÚS, H.; FERRERO, G.; COMAS, J.; RODRIGUEZ-RODA, I. (2009) Comparison of removal of pharmaceuticals in MBR and activated sludge systems. Desalination, v. 250, n. 2, p. 653-659.

SOARES, A.; GUIEYSSE, B.; JEFFERSON, E.; CARTMELL, E.; LESTER, J. N. (2008) Nonylphenol in the environment: A critical review on occurrence, fate, toxicity and treatment in wastewaters. Environment International, v. 34, p. 1033-1049.

SODRÉ, F.F.; LOCATELLI, M.A.F.; JARDIM, W.F. (2010) Occurrence of emerging contaminants in Brazilian drinking waters: a sewage-to-tap issue. Water, Air \& Soil Pollution, v. 206, p. 57-67.

SOUZA, N.C. (2011) Avaliação de Micropoluentes Emergentes em Esgotos e Águas Superficiais. 166 p. Tese (Doutorado em Engenharia Civil) - Universidade Federal do Ceará, Fortaleza, 2011.

SPENGLER, P.; KÖRNER, W.; METZGER, J. (2001) Substances with estrogenic activity in effluents of sewage treatment plants in southwestern Germany. Chemical analysis. Environmental Toxicology and Chemistry, v. 20, n. 10, p. 2133-2141.

STAPLES, C.A.; DORN, P.B.; KLECKA, G.M.; BRANSON, D.R.; O'BLOCK, S.T.; HARRIS, L.R. (1998) A Review of the Environmental Fate, Effects and Exposures of Bisphenol A. Chemosphere, v. 36, p. 2149-2173.

STENSTROM, M.K.; CARDINAL, L.; LIBRA, J. (1989) Treatment of hazardous substances in wastewater treatment plants. Environmental Progress, v. 8, n. 2, p. 107-112.

STUMPF, M.; TERNES, T.A.; WILKEN, R.; RODRIGUES, S.V.; BAUMANN, W. (1999) Polar drug residues in sewage and natural waters in the state of Rio de Janeiro, Brazil. The Science of the Total Environment, v. 255, p. $135-141$

SUÁREZ, S.; CARBALLA, M.; OMIL, F.; LEMA, J. M. (2008) How are pharmaceutical and personal care products (PPCPs) removed from urban wastewaters? Reviews in Environmental Science and Biotechnology, v. 7, p. 125-138

SUMPTER, J.P. (1998) Xenoendocrine disruptors - environmental impacts. Toxicology Letters, v. 102, p. 337-342.

SVENSON, A.; ALLARD, A.; EK, M. (2003) Removal of estrogenicity in Swedish municipal sewage treatment plants. Water Research, v. 37, n. 18, p. $4433-4443$.

TAMBOSI, J.L. (2008) Remoção de fármacos e avaliação de seus produtos de degradação através de tecnologias avançadas de tratamento. 141 p. Tese (Doutorado em Engenharia Química), Universidade Federal de Santa Catarina, Florianópolis, 2008

TAMBOSI, J.L.; YAMANAKA, L.Y.; JOSÉ, H.J.; MOREIRA, R.F.P.M. (2010) Recent Research data on removal of pharmaceuticals from sewage treatment plants (STP). Química Nova, v. 33, p. 411-420.

TAN, B.L.L.; HAWKER, D.W.; MÜLLER, J.F.; LEUSCH, F.D.L.; TREMBLAY, L.A.; CHAPMAN, H.F. (2007) Comprehensive study of endocrine disrupting compounds using grab and passive sampling at selected wastewater treatment plants in South East Queensland, Australia. Environment International, v. 33 , p. 654-669.

TER LAAK, T.L.; DURJAVA, M.; STRUIJS, J.; HERMENS, J.L. (2005) Solid phase dosing and sampling technique to determine partition coefficients of hydrophobic chemicals in complex matrixes. Environmental Science \& Technology, v. 39, n. 10, p. 3736-3742.

TERNES, T.A.; STUMPF, M.; MUELLER, J.; HABERER, K.; WILKEN, R.D.; SERVOS, M. (1999) Behavior and occurrence of estrogens in municipal sewage treatment plants - 1: Investigations in German, Canada and Brazil. Science of the Total Environment, v. 225, p. 80-90.

TERNES, T.A.; HERRMANN, N.; BONERZ, M.; KNACKER, T.; SIEGRIST, H. JOSS, A. (2004) A rapid method to measure the solid-water distribution coefficient $\left(K_{d}\right)$ for pharmaceuticals and musk fragrances in sewage sludge, Water Research, v. 38, p. 4075-4084.

TOXNET. United States National Library of Medice - Toxnet Toxicology Data Network. Hazardous Substances Data Bank. Disponível em: $<$ http://toxnet.nlm.nih.gov/>. Acesso em 30 set. 2011.

USEPA, U.S. Environmental Protection Agency. (2009) Occurrence of Contaminants of Emerging Concern in Wastewater From Nine Publicly Owned Treatment Works. Washington D. C., EUA.

VANDENBERG, L. N.; HAUSER, R.; MARCUS, M.; OLEA, N.; WELSHONS, W. V. (2007) Human exposure to bisphenol A (BPA). Reproductive Toxicology, v. 24, p. 139-177.

VERLICCHI, P.; GALLETTI, A.; AL AUKIDI, M.; PASTI, L.; MARCHETTI, N. (2010) Evaluation of sulphametoxazole, ciprofloxacin and trimethoprim removal in a H-SSF system: An experimental investigation. In: $12^{\text {th }}$ International Conference on Wetland Systems for Water Pollution Control, Proceedings..., Venice: IWA.

VIENO, N.M.; TUHKANEN, T.; KRONBERG, L. (2005) Seasonal Variation in the Occurrence of Pharmaceuticals in Effluents from a Sewage Treatment Plant and in the Recipient Water. Environmental Science \& Technology, v. 39, p. 8220-8226.

VON SPERLING, M. (2002) Princípio do tratamento biológico de águas residuárias: Lodos ativados. 2. ed. Belo Horizonte: Departamento de Engenharia Sanitária e Ambiental, Universidade Federal de Minas Gerais. 428 p.

WENK, J.; Von GUNTEN, U.; CANONICA, S. (2011) Effect of Dissolved Organic Matter on the Transformation of Contaminants Induced by 
Excited Triplet States and the Hydroxyl Radical. Environmental Science and Technology, v. 45, p. 1334-1340.

WHO - World Health Organization. (2002) International Programme on Chemical Safety. Global assessment of the state-of-the-science of endocrine disruptors. Report WHO/PCS/EDC/02.2. Geneva, Suíça, $180 \mathrm{p}$. .

YING, G.; KOOKANA, R.S.; KUMAR, R. (2008) Fate of estrogens and xenoestrogens in four sewage treatment plants with different technologies. Environmental Toxicology and Chemistry, v. 27, n. 1, p. 87-94
ZHANG, Y.; GEIBEN, S.U.; GAL, C. (2008) Carbamazepine and diclofenaco: Removal in wastewater treatment plants and occurrence in water bodies. Chemosphere, v. 73, p. 1151-1161.

ZHANG, Z.; FENG, Y.; GAO, P.; WANG, C.; REN, N. (2011) Occurrence and removal efficiencies of eight EDCs and estrogenicity in a STP. Journal of Environmental Monitoring, v. 13, n. 5, p. 1366-1373.

ZUCCATO, E.; CASTIGLIONI, S.; FANELLI, R.; REITANO, G.; BAGNATI, R.; CHIABRANDO, C.; POMATI, F.; ROSSETTI, C.; CALAMARI, D. (2006) Pharmaceuticals in the environment in Italy: Causes, occurrence, effects and control. Environmental Science and Pollution Research, v. 13, n. 1, p. 15-21. 